\title{
Multichannel Staggered SAR: System Concepts with Reflector and Planar Antennas
}

\author{
Felipe Queiroz de Almeida, Tobias Rommel, Member, IEEE, Marwan Younis, Senior Member, IEEE, \\ Gerhard Krieger, Fellow, IEEE and Alberto Moreira, Fellow, IEEE
}

\begin{abstract}
In the context of radar spaceborne imaging, the novel multichannel Staggered SAR mode represents a promising solution for the mapping of ultra-wide swaths with very high azimuth resolution. This paper extends the analysis of multichannel staggered SAR to planar antenna architectures and provides system design examples both for planar and reflector antenna architectures. Furthermore, a first proof of concept using measured data acquired by an experimental ground-based system is provided to demonstrate the approach's feasibility.
\end{abstract}

Index Terms - Radar, Radar imaging, Spaceborne radar, Synthetic aperture radar, Digital beamforming, High-resolution wide swath

\section{INTRODUCTION}

$\mathrm{S}^{\mathrm{p}}$ PACEBORNE Synthetic Aperture Radar (SAR) [1], [2] images enjoy an increasing acceptance in the scientific community and a growing pool of applications. This is due to the capability of continuous (weather and daylight independent) imaging, as well as to the additional information present in such images, in comparison with other sensors. Maximizing the information content of SAR imagery requires improving the spatial and temporal resolution of acquisitions. This poses the requirement of imaging with high resolution and short-revisit times, which in turn requires the simultaneous acquisition of wide swathes on the ground.

High-resolution wide-swath (HRWS) spaceborne SAR imaging - though beneficial to most applications and bearing the potential to make new applications feasible - presents itself as a challenge in terms of SAR system design. Conventional singlechannel SAR systems [3],[4],[5] are subject to a well-known compromise between the swath width and the maximum achievable azimuth resolution. This prevents them from fulfilling the increasing requirements for continuous high-resolution Earth observation. Such systems are thus no longer an option for stateof-the-art [6] and near-future spaceborne SAR systems. Fig. 1 (a) illustrates a simplified model of the geometry and the echo of a single transmitted pulse (usually a chirp [1]), whereas Fig. 1 (b) shows schematically the timing when a train of pulses is transmitted. In the case under analysis, the pulse repetition

Manuscript received Mai 17, 2017. F. Queiroz de Almeida, T. Rommel, M. Younis, G. Krieger and A. Moreira are with the Microwaves and Radar Institute of the German Aerospace Center (DLR), Oberpfaffenhofen, Germany (e-mail: felipe.queirozdealmeida@dlr.de). frequency (PRF) is too high and thus the echo window length too short to fit the ground echo. This condition illustrates two important effects to which HRWS imaging systems are prone. First, range ambiguities due to the overlap of the end of the echo of a transmitted pulse and the beginning of the echo of the next pulse (cf. red and blue echoes in Fig. 1 (b)). Second, blind ranges, which arise due to the fact that, in a monostatic system, the received signal cannot be recorded during transmission (cf. dashed portions of echoes in the same figure). As seen in Fig. 1 (b), this creates a gap in the receive echo.

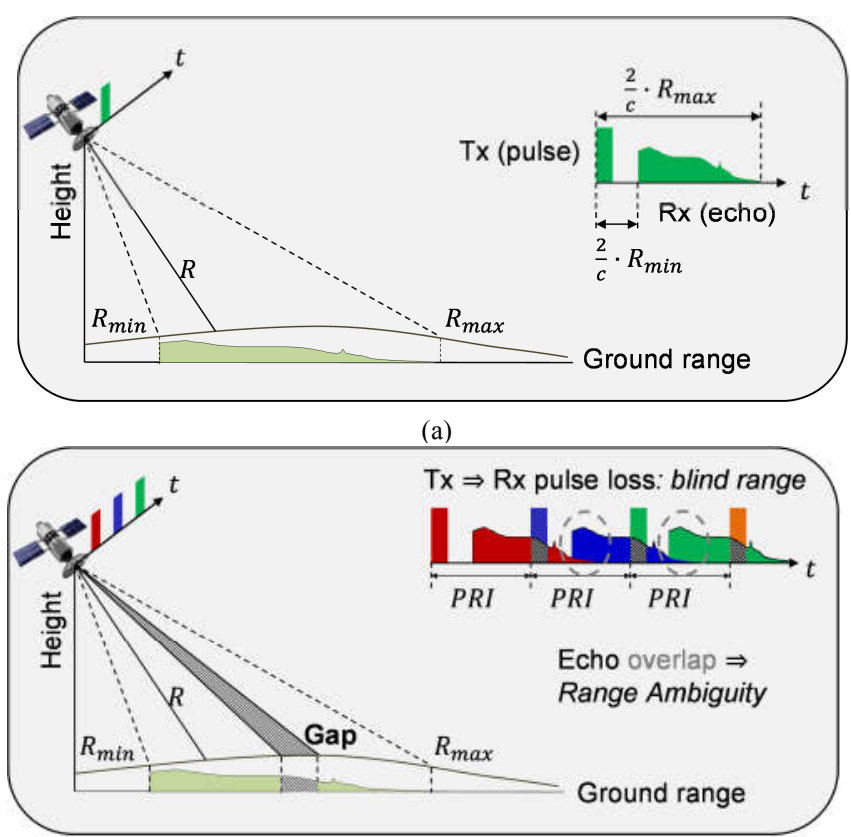

(b)

Fig. 1: Schematic representation of swath geometry. (a) Transmitted pulse and the echo of an arbitrary swath of interest. (b) Timing for the transmission of a train of pulses with a PRF which is too high to yield unambiguous imaging of the whole swath, though suitable for the support of a fine azimuth resolution. Blind ranges and range ambiguities are seen to arise.

Research in this field [3],[4],[7],[8],[9],[10],[11],[12] identifies the usage of multiple receiver channels as a key technology in enhancing system performance to the required 
levels. The resulting Single-Input Multiple-Output ${ }^{1}$ (SIMO) systems may have multiple output channels both in the elevation and azimuth dimensions. These systems require suitable digital beamforming $(\mathrm{DBF})$ techniques for processing of the data.

Additional output channels in elevation lead to systems capable of performing, e.g., SCORE (SCan-On-Receive [14]) with multiple simultaneous elevation beams [9],[10],[15],[16]. These systems achieve imaging of several sub-swaths simultaneously, increasing the swath width for a given PRF while distinguishing between range ambiguous returns by means of their different angles of arrival in elevation. This capability is the key to extend the swath beyond the limits imposed by the non-ambiguous receive interval (cf. Fig. 2 (a)), effectively decoupling the duration of the unambiguous receive window from the PRF.

This, however, does not solve the problem of the blind ranges: Note that in Fig. 2 (a) the gaps are still present. A possible solution is a cyclical variation of the system's pulse repetition interval (PRI), which is known as staggered SAR [17],[18]. Blockage of the receive signal by transmission events still occurs, but as long as the PRI variation is fast enough (cf. [11],[18],[19]), the position of the blocked ranges is different for each pulse and has no overlap in azimuth with neighboring pulses, as shown in Fig. 2 (b). It should be stressed that, in a constant PRF system, the gaps (i.e. ranges lost due to transmission events) are at the same position for every transmitted pulse, and thus no information regarding these ranges is available in the synthetic aperture, leading to the occurrence of the blind range. In contrast, as a consequence of the PRI staggering, the blocked ranges are distributed over range and become azimuth dependent. Recovery is nonetheless possible by interpolation over azimuth from neighboring pulses. Hence, even though the effect of Tx blockage is still present, blind ranges (characterized by no recorded signal for every pulse) do not occur.

Such techniques allow very wide swaths to be imaged, but the best achievable azimuth resolution is limited if only a single channel in azimuth is used. For instance, Tandem-L [6] achieves using this technique a $7 \mathrm{~m}$ azimuth resolution over a $350 \mathrm{~km}$ swath. The HRWS imaging capability of this class of system can be further extended by the use of multiple receive channels in azimuth, leading to the multichannel (in azimuth) staggered SAR concept, which will be described in more detail in the body of the paper.

The use of multiple receivers in the azimuth or along-track dimension with a constant $\mathrm{PRF}^{2}$ is a different approach towards HRWS. The main goal is to increase the sampling rate and generate an equivalent single-channel output which can be processed by existing SAR image focusing algorithms.

${ }^{1}$ Multiple-Input Multiple-Output (MIMO) systems [13] can in principle be realized by adaptation of SIMO hardware architectures in combination with a set of properly designed transmit waveforms and additional processing steps. In this sense, the schemes proposed for SIMO architectures are also of interest for MIMO applications, observing some peculiarities of the latter.

${ }^{2}$ Note that SAR systems are typically capable of changing their PRF from acquisition to acquisition or from sub-swath to sub-swath. The constant PRF constraint refers to the observation time within a given synthetic aperture.

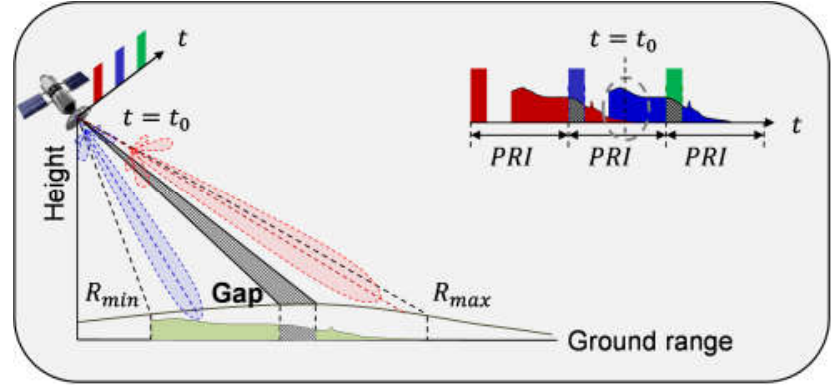

(a)

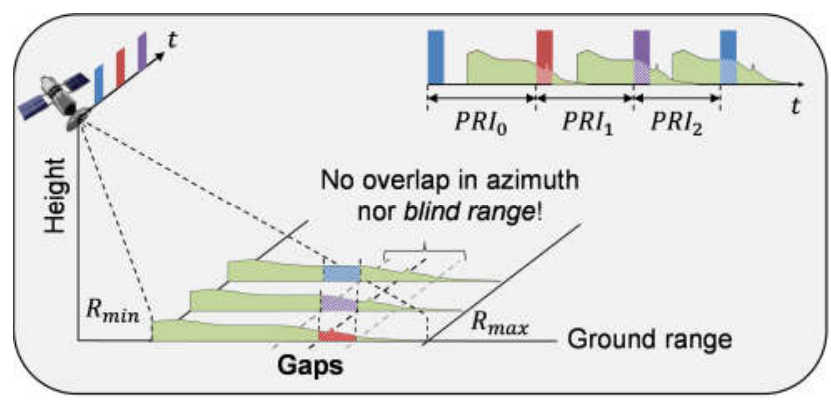

(b)

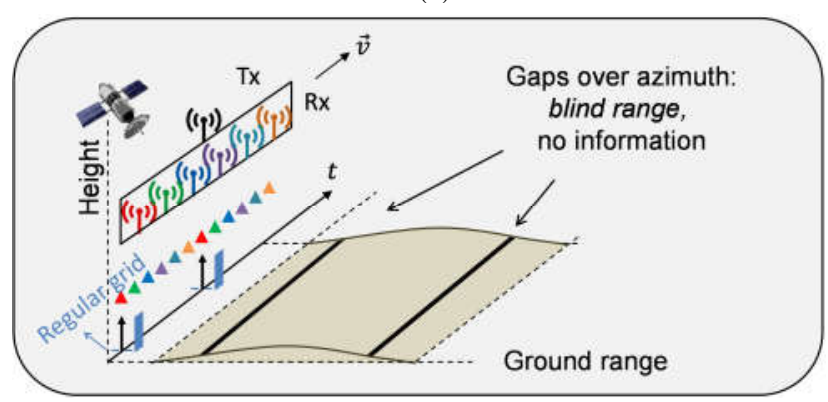

(c)

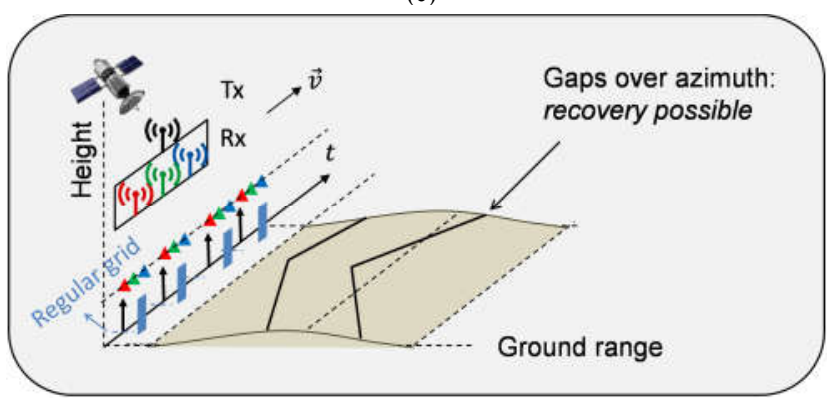

(d)

Fig. 2: Schematic representation of HRWS geometry and imaging modes. (a) System with constant PRI - and thus prone to blind ranges - but multiple elevation beams, which resolves the range ambiguities. (b) Staggered SAR system, in which the different PRIs cause the transmission-induced blockage to affect different ranges for each pulse without overlap in azimuth; a posterior interpolation and recovery allows a gapless acquisition over the swath. (c) Multichannel system in azimuth with a constant PRI. The Tx pulses are shown over the slow time axis for comparison with the previous figures, and the phase center position of the transmitted and received pulses are represented by arrows and triangles, respectively, illustrating the sampling across the aperture. Use of multiple channels allows forming a regular grid with a gain in the sampling rate corresponding to the number of channels, thus enabling adequate sampling in spite of a lower PRF. Blind ranges however (dark lines on ground) occur. (d) Use of multiple channels with PRI staggering and the consequent formation of a non-uniform grid for the received pulses, whereas avoiding blind ranges. 
The rationale is closely related to the Displaced Phase Center Array (DPCA) technique [3],[4] and is illustrated in Fig. 2 (c). Multiple receivers allow gathering additional samples for each transmitted pulse. This achieves a decoupling of the sampling rate of the signal in azimuth from the PRF. Thus, a lower PRF can be used, while keeping a high sampling rate. The low PRF allows a wider unambiguous receive interval and thus a wider swath. The high sampling rate, in turn, allows the system to support a high azimuth resolution. In practice, as discussed in [3], this technique is very effective at improving the azimuth resolution at a given PRF. However, it may lead to unrealistically long antenna sizes (several tens of meters), if the required swath is too wide (a few hundreds of kilometers). In practice, very high resolution may be achieved, but at narrower swaths, e.g., $1 \mathrm{~m}$ resolution over $70 \mathrm{~km}$ for HRWS [20]. Moreover, this class of system typically employs frequency domain DBF techniques. This prevents a straightforward combination with the staggered PRI technique, which uses nonuniform temporal sampling.

Both of the aforementioned techniques present limitations. Single-channel staggered SAR has a limited azimuth resolution, whereas multichannel systems in azimuth show limited swath for realistic antenna sizes, and are constrained to operate with a constant PRF. The novel multichannel staggered SAR modes and processing algorithms - introduced in [21], [22], [23] - present the opportunity to overcome these limitations and combine the strengths of both systems. The resulting concept presents multiple elevation beams and PRI staggering on one hand and multiple channels in azimuth on the other (cf. Fig. 2 (d)). This allows HRWS system designs with the capability of outperforming systems using only one of the two techniques, achieving very demanding HRWS imaging requirements with a feasible antenna size. [22] provides a thorough description of the modeling and processing strategy for a quad-polarization reflector antenna system design example. This paper builds on it by discussing various further aspects of the methods. Firstly, a theoretical analysis for planar antenna systems, describing the required design changes, is provided. Additionally, an example of a reflector system design in single-pol is included for comparison. It also illustrates how in this less demanding scenario the resolution and swath width trade-off can be pushed even further, to reach an unprecedented $1 \mathrm{~m}$ azimuth resolution in a $500 \mathrm{~km}$ wide swath. Finally, a proof of concept of the method with reflector antennas is provided, based on measurement data acquired by an experimental ground-based multichannel radar system. It is interpolated to simulate a staggered acquisition and then reconstructed using the discussed techniques.

\section{A. Paper Structure}

The rest of this paper is organized into two parts, separating the material referring to reflector antennas and that related to planar ones. The analogy and interchangeability between these classes of antennas should, however, be kept in mind. Within Part I, Section II provides a brief summary of the azimuth resampling techniques discussed in [22]. Section III provides an additional example of a reflector system design, this time in single polarization. Section IV contains material on an experiment-based proof of concept, employing a reflector antenna with a multichannel feed in X-band. Part II starts with Section V, which turns focus to a theoretical analysis of planar antenna systems. It examines the changes in the sampling configuration introduced by this class of antenna, as well as how the design should be adapted to reflect their physical characteristics. Section VI provides a design example illustrating the discussed aspects. Last, the content of both parts is reviewed and summarized in Section VII, which also contains a comparison between the two classes of antennas in the context of multichannel staggered SAR systems.

\section{PART I: REFLECTOR ANTENNA SYSTEMS}

\section{Multichannel Staggered SAR Azimuth BEAMFORMING: VIRTUAL BEAM SYNTHESIS TECHNIQUES}

Multichannel Staggered SAR systems acquire data which are sampled in azimuth in a periodically non-uniform manner (due to the PRI staggering). The acquisition takes place over multiple channels with, as a rule, different antenna patterns. A resampling step is therefore necessary to convert from this input (multichannel) grid to a uniform one. This output grid should moreover be equivalent to the one acquired by a single-channel system sampled at a higher rate, with a stable azimuth pattern, so that it can be further processed using conventional SAR processing algorithms.

The resampling problem is illustrated schematically in Fig. 3. The sampling instants of the periodically non-uniform staggered SAR signal (assumed to have $N_{\text {eff }}$ samples per cycle, after accounting for Tx-induced blockage at a specific range) is represented by the arrows in the first plot. The system has $N_{c h}$ azimuth channels with known antenna patterns, and the triangles with different colors represent the samples from each of them. Given the knowledge of the sampling and the antenna patterns, the task is to determine the weights to resample the signal to a uniform grid. Specifically, an optimization criterion for the weights needs to be defined. This is implemented so that the samples from $N_{p}$ pulses over all $N_{c h}$ channels should be combined to approximate each of the $N_{c h} \cdot N_{\text {eff }}$ samples of a regular grid spanning the cycle

$$
v_{m}\left(f_{d}\right)=G_{k_{1}[m]}\left(f_{d}\right) \cdot \exp \left(-j \cdot 2 \cdot \pi \cdot t_{R X}\left[k_{2}[m]\right] \cdot f_{d}\right),
$$

duration. The solution is implemented as a combination of a digital filter $^{3}$ over the pulses and a conventional complex-weight beamformer ${ }^{4}$ over the channels at each pulse position, combining $N_{\text {win }}=N_{p} \cdot N_{c h}$ input samples for the estimation of each output sample. The resampled output is approximately ${ }^{5}$ regularly sampled at a rate of $P R F_{\text {multi }}=N_{c h} \cdot \overline{P R F}_{\text {eff }}$, where $\overline{P R F}_{\text {eff }}$ is the effective average sampling rate $^{6}$ of the pulses, considering blockage. This means that both the regularization of the sample instants and the increase of the sampling rate by a factor equal to

\footnotetext{
${ }^{3}$ The filter structure is a convolution over a finite window without feedback from previous outputs. This is similar to a Finite Impulse Response (FIR) except for the non-uniform sampling instants.

${ }^{4}$ The general framework of DBF in complex domain is assumed. As analyzed in [22], an alternative is an architecture with time varying real-weighted beamforming coefficients applied to the intermediate frequency (IF) data streams, coupled with a modified quadrature demodulation. It leads to equivalent results and has the potential of saving resources in an actual implementation, at least for planar antennas.

${ }^{5}$ Note that the resampling is optimal in the sense of the adopted criterion. Residual errors do, however, remain, meaning the output is not exactly regular.

${ }^{6} \overline{P R F}_{\text {eff }}$ is range-dependent according to the timing of the PRI sequence, as the number of non-blocked pulses $N_{\text {eff }}$ changes. This means that an interpolation stage to a common sampling rate across the swath is necessary [22].
} 
the number of channels are done simultaneously by the proposed processing framework.

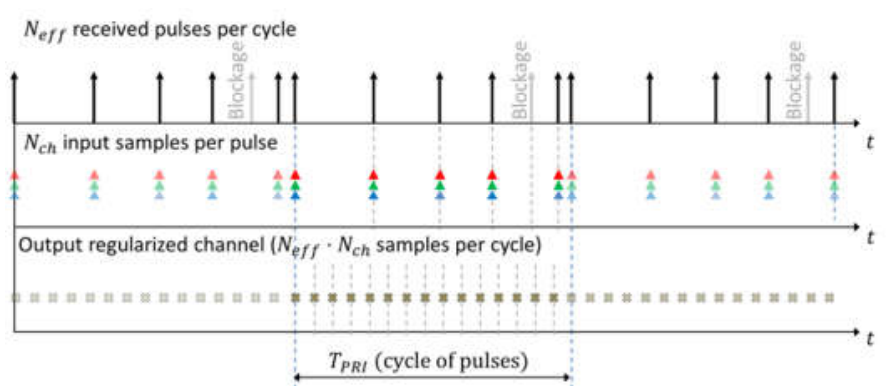

(a)

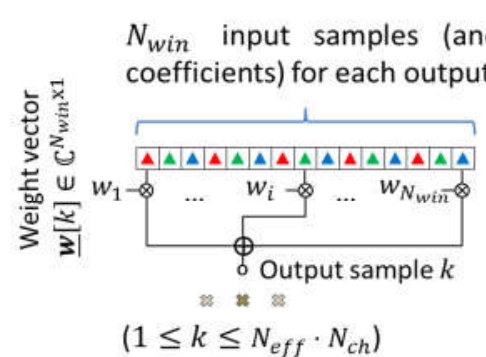

(b)

Fig. 3: Required resampling operation focusing on a cycle of pulses of duration $T_{P R I}$. (a) Input grid composed of a set of $N_{e f f}$ staggered PRI pulses received by an $N_{c h}$ channel configuration yielding in total $N_{e f f} \cdot N_{c h}$ samples per cycle; these samples are to be rearranged uniformly in the output grid, which is equivalent to a single channel system at a higher sampling rate. (b) Formation of a single sample, index $k$, of the output grid, which is derived from the combination of $N_{\text {win }}$ input samples with complex weights.

The Virtual Beam Synthesis (VBS) algorithm, explained in [22],[23] in detail, gives a solution to this problem. The reader is referred to [22] for an in-depth description of the technique (originally motivated by the properties of a reflector antenna) and a discussion of its implications for system design and performance. The purpose of this section is to summarize the main aspects of the method, serving as basis to the extension for planar antennas considered in Section V.

The solution involves first modeling the inputs in the Doppler frequency $f_{d}$ domain. An extended array manifold vector $\boldsymbol{v}\left(f_{d}\right)$ of dimension $N_{\text {win }}$ is formed to account both for the azimuth patterns $G_{n}\left(f_{d}\right), 1 \leq n \leq N_{c h}$, and the recorded pulse time instants $t_{R X}[i], 1 \leq i \leq N_{p}$. It is denoted as for $1 \leq m \leq N_{\text {win }}$, with

$k_{1}[m]=\left((m-1) \bmod N_{c h}\right)+1$

$k_{2}[m]=(m-1) \% N_{c h}+1$,

where $\bmod$ and $\%$ denote the remainder and quotients of integer division, respectively. Note that the choice of the number of pulses $N_{p}$ to include in the manifold allows modeling arbitrary resampling strategies ${ }^{7}$.

\footnotetext{
${ }^{7}$ In [35], a beam steering technique for a reflector with a multichannel feed is proposed for staggered SAR sample regularization. It can be considered a particular case of this formulation for $N_{p}=1 \Rightarrow N_{\text {win }}=N_{c h}$, meaning a
}

The next step is to model the desired output samples as a regular grid described by a set of $N_{\text {out }}=N_{c h} \cdot N_{\text {eff }}$ output patterns $G_{G O A L}\left(f_{d}, k\right)$. Due to the cyclical nature of the sampling, it suffices to describe one pattern for each sample $1 \leq k \leq N_{\text {out }}$. The patterns may be written as

$G_{G O A L}\left(f_{d}, k\right)=G_{c o m}\left(f_{d}\right) \cdot \exp \left(-j \cdot 2 \cdot \pi \cdot t_{\text {out }}[k] \cdot f_{d}\right)$,

where the uniformity of the grid is imposed by setting

$t_{\text {out }}[k]=\frac{k-1}{N_{c h} \cdot \overline{P R F}_{\text {eff }}}+\delta t$,

with $\delta t$ being a degree of freedom [22]. $G_{\text {com }}\left(f_{d}\right)$ is the desired common pattern of the equivalent single-channel system which the multichannel staggered SAR system seeks to emulate after the beamforming. A meaningful (physically implementable) choice for $G_{c o m}\left(f_{d}\right)$ is necessary for adequate performance. The sum pattern

$G_{\text {sum }}\left(f_{d}\right)=\frac{1}{\sqrt{N_{c h}}} \cdot \sum_{i=1}^{N_{c h}} G_{i}\left(f_{d}\right)$,

is the default choice for a reflector system and the case of planar arrays will be discussed in Section V.

In the VBS method, the $N_{w i n}$-element complex weight vector $\boldsymbol{w}$ is obtained by minimizing a cost function $\bar{\xi}\left(G_{G O A L}\left(f_{d}, k\right), v_{m}\left(f_{d}\right)\right)$, a fairly general case of which is

$\xi_{J}=(1-\alpha) \cdot \frac{\xi_{M S E}}{n_{M S E}}+\alpha \cdot \frac{n_{S N R}}{\xi_{S N R}}$,

where $n_{M S E}$ and $n_{S N R}$ are normalization factors and $\alpha$ is an SNR-sensitivity parameter in the interval $[0,1]$. It is chosen as part of the weight design to emphasize either

$\xi_{M S E}=\int_{f_{1}}^{f_{2}}\left(G_{G O A L}\left(f_{d}\right)-\underline{\boldsymbol{w}}^{\boldsymbol{H}} \cdot \underline{\boldsymbol{v}}\left(f_{d}\right)\right)^{2} d f_{d}$

or

$\xi_{S N R}=\frac{\int\left|\underline{\boldsymbol{w}}^{H} \cdot \underline{\boldsymbol{v}}\left(f_{d}\right)\right|^{2} d f_{d}}{\underline{\boldsymbol{w}}^{H} \cdot \underline{\boldsymbol{w}}}$

The first component $\xi_{M S E}$ measures the quality of the goal pattern (regular grid) implementation using the weights ${ }^{8} \underline{w}$ within a given bandwidth, meaning a low $\alpha$ leads to improved regularity and better ambiguity suppression. Conversely, $\xi_{S N R}$ is a form of normalized SNR measurement with respect to white noise, and a higher $\alpha$ improves the SNR scaling of the beamformer with respect to the inputs.

single pulse is used for each output sample. The mapping between primary beam steering angle and secondary pattern phase shift is an indirect form of solving the weight problem, tackled here by optimization of a cost function.

${ }^{8} \underline{\boldsymbol{w}}$ or $\underline{\boldsymbol{w}}[k]$ is here a complex weight vector of dimension $N_{\text {win }}$, used for the implementation of a given output sample $k, 1 \leq k \leq N_{\text {out }}$, in a cycle of the output grid. The two notations are used interchangeably, and the dependency on $k$ is omitted whenever possible to simplify the formulas, but should be kept in mind. 
The solution to the minimization of (6) [22], [25] is the nonlinear equation ${ }^{9}$

$$
\begin{aligned}
& \frac{1-\alpha}{n_{M S E}} \cdot\left(\underline{\boldsymbol{R}}_{v} \cdot \underline{\boldsymbol{w}}-\underline{\boldsymbol{\sigma}}_{G}[k]\right)+\alpha \cdot n_{S N R} \cdot \\
& \frac{\left(\underline{\boldsymbol{w}}^{H} \cdot \underline{\boldsymbol{R}}_{v} \cdot \underline{\boldsymbol{w}}\right) \cdot \underline{\boldsymbol{w}}-\left(\underline{\boldsymbol{w}}^{H} \cdot \underline{\boldsymbol{w}}\right) \cdot\left(\underline{\boldsymbol{R}}_{v} \cdot \underline{\boldsymbol{w}}\right)}{\left(\underline{\boldsymbol{w}}^{H} \cdot \underline{\boldsymbol{R}}_{\boldsymbol{v}} \cdot \underline{\boldsymbol{w}}\right)^{2}}=\underline{\mathbf{0}},
\end{aligned}
$$

where

$\underline{\boldsymbol{\sigma}}_{G}[k] \triangleq \int G_{G O A L}^{*}\left(f_{d}, k\right) \cdot \underline{\boldsymbol{v}}\left(f_{d}\right) d f_{d}$

is the cross-covariance between the goal pattern and the manifold and

$\underline{\boldsymbol{R}}_{\boldsymbol{v}} \triangleq \int \underline{\boldsymbol{v}}\left(f_{d}\right) \cdot \underline{\boldsymbol{v}}\left(f_{d}\right)^{H} d f_{d}$

is the manifold's auto-covariance matrix. (9) can be solved numerically exploiting the closed form solutions for the particular cases in which $\alpha=0$ (leading to a linear system of equations with solution $\underline{\boldsymbol{w}}=\underline{\boldsymbol{R}}_{v}^{-1} \cdot \underline{\boldsymbol{\sigma}}_{G}[k]$ ) and $\alpha=1$ (which leads to an eigenvector problem $\gamma \cdot \underline{\boldsymbol{w}}=\underline{\boldsymbol{R}}_{\boldsymbol{v}} \cdot \underline{\boldsymbol{w}}$, with the scalar eigenvalue $\gamma$ representing the maximum $\xi_{S N R}$ ) as first guesses.

The most important quality measures of a given output grid are the achieved Mean Squared Error (MSE) in (7) and the SNR scaling with respect to the common pattern $G_{c o m}$. The latter may be defined as

$\Phi_{S N R}=\sum_{k} \frac{\left.\int \underline{\underline{w}}[k]^{H} \cdot \underline{\boldsymbol{v}}\left(f_{d}\right)\right|^{2} d f_{d}}{\int\left|G_{\text {com }}\left(f_{d}\right)\right|^{2} d f_{d}} \cdot \frac{1}{\underline{\boldsymbol{w}}[k]^{H} \cdot \underline{\boldsymbol{w}}[k]}$,

in which each term in the summation is proportional to the sample's antenna pattern's gain within the processed bandwidth. The summation implements an average over the output grid. The normalization to the mean gain of the common pattern allows this quantity to be interpreted as the SNR scaling in comparison to a single-channel system possessing exactly the pattern which the resampling seeks to approximate.

\section{Simulation Results FOR REFLECTOR ANTENNA}

This section provides an example of a single-polarization multichannel staggered SAR mode aimed at a very fine $1.0 \mathrm{~m}$ azimuth resolution over a very wide $500 \mathrm{~km}$ swath in L-band. The adopted swath width is enough to provide a global coverage in less than 6 days. These represent very demanding HRWS requirements that currently cannot be achieved by any other known singlesatellite system concept with a feasible antenna size. The main image quality requirement guiding the design is to provide azimuth ambiguity-to-signal ratio (AASR), range ambiguity-to-signal ratio (RASR) and noise-equivalent sigma zero (NESZ) levels better than

\footnotetext{
${ }^{9}$ The azimuth interpolation technique in

[11],[18], has weight values proportional to the cross-correlation vector between output and input sampling instants and the inverse of the input grid auto-correlation matrix. This approach is conceptually analogous to the case $\alpha=0$ for a single-channel system in azimuth $\left(N_{c h}=1\right)$. The main difference is that in [11],[18] an analytical correlation model is used for a uniformly illuminated aperture to estimate the window length $N_{\text {win }}=N_{p}$ and the correlations involved, instead of using the pattern information as input.
}

$-25 \mathrm{~dB}$. Note that the spectral allocation standards [26] at L-band limit the pulse bandwidth to $85 \mathrm{MHz}$, meaning the maximum ground range resolution is about $4.5 \mathrm{~m}$, for a minimum incidence angle of $25^{\circ}$.

The performance analysis is thus presented in terms of these parameters. The AASR estimation follows the strategy in [27], based on the difference of the integral of the impulse responses of the reconstructed signal and that of an alias-free reference, simulated with uniform sampling over azimuth. The RASR estimation employs the integration of the $2 \mathrm{D}$ antenna patterns and follows the staggered-SAR range ambiguity model of [27], taking furthermore into account the increased sampling of the multichannel case. The average L-band backscatter model is that of [28]. The effect echoes from nearly normal incidence is addressed by showing, for each system, the range ambiguity levels both with and without their power contribution. A worstcase analysis of the coherent (specular) [29] returns near nadir (cf. A.IV) is also provided in terms of the Nadir Return-to-Signal Ratio (NRSR). The relevance of this contribution is determined mostly by the antenna gain at nadir. Reflector systems, owing to their directivity, are thus less affected (the contribution may be noticeable for planar antennas, as will be discussed in Part II). Another relevant aspect in the case of staggered PRI is that the power of the nadir returns is smeared over azimuth, as the range bins affected by them change with slow time. This effect leads to a further attenuation of the power of range-ambiguous returns at a given range. Finally, the NESZ estimation follows the modeling of [30] for a reference system with the pattern $G_{\text {com }}\left(f_{d}\right)$ in azimuth. The result is degraded by the SNR scaling $\Phi_{S N R}$ (cf. (12)) with respect to that pattern. As will be shown, $G_{\text {com }}$ is different for reflector and planar antenna systems.

The reflector and feed design has the one presented in [15] as a starting point, adapted as described below. [22] shows a first example of a reflector system using the technique, but at quadpol and with less demanding performance requirements. The antenna system and the mode's operational characteristics are described in TABLE I. The feed architecture in azimuth has doublets spaced by $0.6 \lambda$ combined pairwise to form channels with $1.2 \lambda$ spacing, and the number of azimuth channels is increased (in comparison to [15]) to 9, to cover the higher Doppler bandwidth required by the azimuth resolution.

The PRI sequence shows a linear PRI variation according to

$P R I_{n}=P R I_{0}-n \cdot \Delta$

for $0 \leq n \leq N_{P R I}-1$ (and then repeated cyclically). It is designed following the fast PRI variation criterion of [11]. It ensures that, at any given range within the swath, two consecutive samples of a given azimuth channel are never lost. Note that, in the multichannel case, whenever a blockage event due to Tx occurs, the samples from all azimuth receive channels are lost at the same time.

The patterns (directivity) are simulated using the GRASP software [31]. Patch feed antennas with radiation patterns following the model of [32] are assumed. The effect of blockage of the incident waves over the reflector's surface due to the feed (its "shadow") is taken into account. 
TABLE I

SingLE-POL SIMULATION SCENARIO PARAMETERS

\begin{tabular}{|c|c|c|}
\hline \multicolumn{3}{|c|}{ Platform and Swath Parameters } \\
\hline Quantity & Symbol & Value \\
\hline Orbit height & $\overline{h_{\text {orbit }}}$ & $800 \mathrm{~km}$ \\
\hline Swath width on ground & $W_{\text {ground }}$ & $500 \mathrm{~km}$ \\
\hline $\begin{array}{l}\text { Swath minimum/maximum } \\
\text { (off-nadir) look angle }\end{array}$ & $\phi_{\min } / \phi_{\max }$ & $20.3^{\circ} / 43.1^{\circ}$ \\
\hline \multicolumn{3}{|c|}{ Reflector and Feed Parameters } \\
\hline Quantity & Symbol & Value \\
\hline Diameter & $D$ & $15.0 \mathrm{~m}$ \\
\hline Focal length & $F$ & $15.0 \mathrm{~m}$ \\
\hline Feed offset in elevation & $d_{O F F}$ & $10.0 \mathrm{~m}$ \\
\hline Center frequency & $f_{0}$ & $1.2575 \mathrm{GHz}$ \\
\hline $\begin{array}{l}\text { Number of channels in } \\
\text { elevation/azimuth }\end{array}$ & $N_{e l} / N_{a z}$ & $55 / 9$ \\
\hline Channel spacing in elevation/azimuth & $d_{e l} / d_{a z}$ & $0.6 \lambda / 1.2 \lambda$ \\
\hline Elevation tilt angle & $\phi_{\text {tilt }}$ & $32.5^{\circ}$ \\
\hline Feed losses & & $2.0 \mathrm{~dB}$ \\
\hline Feed dimensions (elevation, azimuth) & $\left(L_{e l}, L_{a z}\right)$ & $(7.88,2.57) \mathrm{m}$ \\
\hline \multicolumn{3}{|c|}{ Pulse and $\mathrm{T} x / \mathrm{Rx}$ Hardware Parameters } \\
\hline Quantity & Symbol & Value \\
\hline Average PRF (both polarizations) & $\overline{P R F}$ & $2120 \mathrm{~Hz}$ \\
\hline Initial PRI & $P R I_{0}$ & $503.5 \mu \mathrm{s}$ \\
\hline $\begin{array}{l}\text { PRI sequence step (between pulses of } \\
\text { same polarization) }\end{array}$ & $\Delta$ & $-2.36 \mu \mathrm{s}$ \\
\hline PRI sequence length & $N_{P R I}$ & 28 \\
\hline Pulse duty cycle & $T_{d c}$ & $6 \%$ \\
\hline Pulse (chirp) bandwidth & $B w_{\text {chirp }}$ & $85 \mathrm{MHz}$ \\
\hline $\begin{array}{l}\text { Peak transmit power of a } \mathrm{Tx} / \mathrm{Rx} \\
\text { Module }\end{array}$ & $P_{T X}$ & $87.0 \mathrm{~W}$ \\
\hline Average transmit power & $\bar{P}_{T X}$ & $2583.90 \mathrm{~W}$ \\
\hline System noise temperature & $T_{\text {noise }}$ & $649 \mathrm{~K}$ \\
\hline Transmitted polarizations & - & $\mathrm{V}$ \\
\hline
\end{tabular}

\begin{tabular}{lll}
\hline \hline \multicolumn{3}{c}{ Processing Parameters } \\
\hline Quantity & Symbol & Value \\
\hline Goal azimuth resolution & $\delta_{a z}$ & $1 \mathrm{~m}$ \\
Processed Doppler bandwidth & $B w_{\text {proc }}$ & $7504 \mathrm{~Hz}$ \\
$\begin{array}{l}\text { Number of simultaneous elevation } \\
\text { beams }\end{array}$ & $N_{\text {beams }}$ & 5 \\
$\begin{array}{l}\text { Elevation beamforming sidelobe } \\
\text { constraint }\end{array}$ & $20 \cdot \log _{10}\left(\epsilon_{S D L}\right)$ & $-36 \mathrm{~dB}$ \\
$\begin{array}{l}\text { Number of samples in azimuth } \\
\text { beamformer window }\end{array}$ & $N_{\text {win }}$ & 45 \\
SNR emphasis parameter & $\alpha$ & 0.5 \\
\hline \hline
\end{tabular}

The DBF processing of the data starts over the elevation channels. The goal is to form a high gain SCORE beam for each range. The sidelobe-constrained beamformer described in Appendix A is employed to combine 9 elevation elements at a time so that the sidelobes within the area generating rangeambiguous returns are $36 \mathrm{~dB}$ below the main beam.

Following the elevation beamforming, azimuth beamforming according to the strategy of Section II is applied, with $\alpha=0.5$. In light of the large number of azimuth channels, the pulse vicinity of the extended manifold was limited to 5 pulses, so that $N_{\text {win }}=5 \cdot 9=45$ input samples are used to form each output sample.
The performance in terms of the AASR, RASR and NESZ is depicted over the swath in Fig. 4, alongside the azimuth resolution.

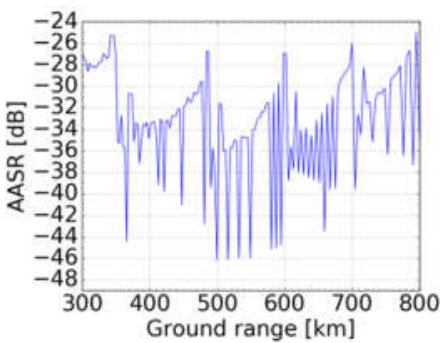

(a)

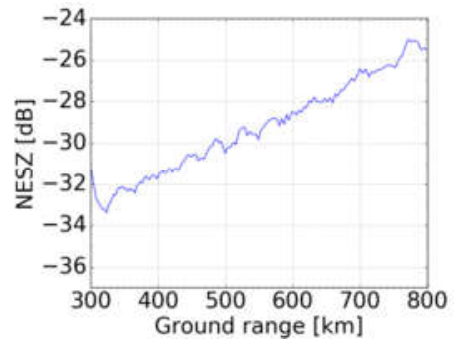

(c)

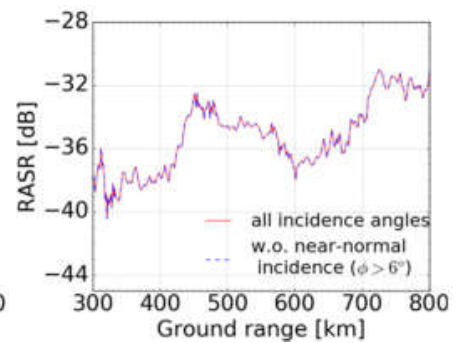

(b)

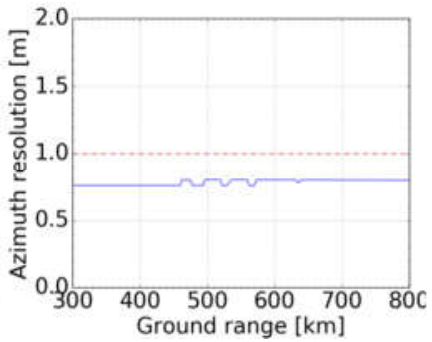

(d)

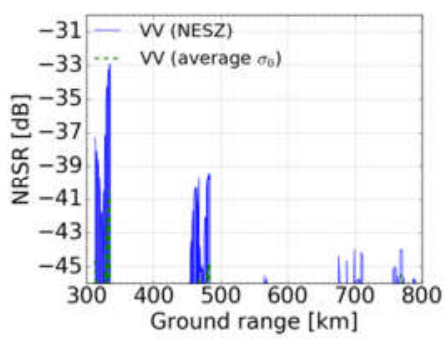

(e)

Fig. 4: Key performance parameters over swath for single-pol high-resolution mode of reflector system: (a) AASR, (b) RASR with (red) and without (blue) the contribution of nadir returns, (c) NESZ, (d) azimuth resolution and (e) NRSR both with respect to NESZ (noise floor) and average $\sigma_{0}$, below $-30 \mathrm{~dB}$ in this case.

In this example, the azimuth resolution is better than 0.8 $\mathrm{m}$ over the swath. The goal of $1.0 \mathrm{~m}$ is achieved, with margin for spectral weighting. The AASR is better than $25.0 \mathrm{~dB}$ and the RASR better than $-31.0 \mathrm{~dB}$. The RASR levels with and without the contribution of nadir returns are seen to be virtually the same. This is due to the reflector pattern's directivity (very low gain at nadir) combined with the attenuation induced by the staggering. The NESZ is better than $-25.0 \mathrm{~dB}$. The NRSR (cf. A.IV) describes the power of worst-case coherent nadir returns with respect to the signal, both at the NESZ (noise floor) and the average backscatter level. Since both levels are under $-30 \mathrm{~dB}$, no artifacts in the image are expected.

\section{EXPERIMENTAL PROOF OF CONCEPT WITH REFLECTOR ANTENNA SYSTEM}

In the interest of allowing experimental demonstrations of novel DBF techniques, an experimental multichannel radar system was developed and built at the Microwaves and Radar Institute of DLR [33],[34]. The system shows great flexibility and also allows the 
usage of reflector antennas for DBF [35]. In this section, a proof of concept of the beamforming technique of Section II using this system is presented. Section IV.A describes the system parameters and the experimental setup. Section IV.B explains the data processing chain and the adopted calibration procedures. Finally, Section IV.C presents and discusses the results.

\section{A. The Ground-Based Demonstrator and the Experimental Setup}

The experimental data take was performed with a reflector antenna configuration. The system employs an X-band reflector antenna with 8 azimuth feed elements on receive, whereas a separate horn antenna is used for transmit. The most important system parameters are summarized in TABLE II.

TABLE II

DEMONSTRATOR SYSTEM AND EXPERIMENT PARAMETERS

\begin{tabular}{lll}
\hline \multicolumn{3}{c}{ Antenna, Pulse and Tx/Rx Hardware Parameters } \\
\hline \hline Quantity & Symbol & Value \\
\hline Center frequency & $f_{0}$ & $9.58 \mathrm{GHz}$ \\
Chirp bandwidth & $B W_{\text {chirp }}$ & $300 \mathrm{MHz}$ \\
Intermediate center frequency & $f_{I F}$ & $205 \mathrm{MHz}$ \\
Peak output power & $P_{T x}$ & $18 \mathrm{dBm}$ \\
ADC sampling rate (real data) & $f_{S}$ & $1 \mathrm{GS} / \mathrm{s}$ \\
ADC resolution & $r_{A D C}$ & $10 \mathrm{bit}$ \\
Elliptical reflector major axis & $D_{\text {maj }}$ & $1.0 \mathrm{~m}$ \\
Elliptical reflector minor axis & $D_{\text {min }}$ & $0.7 \mathrm{~m}$ \\
Reflector focal length & $F_{r e f}$ & $0.5 \mathrm{~m}$ \\
Reflector offset in elevation & $O_{r e f}$ & $0.35 \mathrm{~m}$ \\
Feed element (horn antenna) spacing & $d_{a z}$ & $4.4 \mathrm{~cm}$ \\
Pulse length & $T_{P}$ & $10.0 \mu \mathrm{s}$ \\
System PRF & $P R F$ & $10.0 \mathrm{~Hz}$ \\
Transmitted/Received polarization & - & $\mathrm{VV}$ \\
No. of channels in elevation/azimuth & $N_{e l} / N_{a z}$ & $1 / 8$ \\
\hline \hline
\end{tabular}

As seen in Fig. 5 (a), the imaged scene consists of a calibration corner reflector, plus additional targets (a formation of four other corner reflectors and a metal wire fence). As visible in more detail in Fig. 5 (b), the antennas were mounted atop a $6.34 \mathrm{~m}$ mast and carried by a rail car, propelled by a step motor at the constant velocity of $8.5 \mathrm{~cm} / \mathrm{s}$. Fig. 5 (c) shows the calibration corner reflector placed at the end of the sandbox. In the image, the position of the corner is marked to improve visibility.

Fig. 5 (d) shows a close-up of the Rx reflector with its multichannel feed (the tripod is however not part of the experimental configuration). The feed system itself with the 8 horn antennas is visible in Fig. 5 (e). Its outputs are individually digitized, constituting 8 channels over azimuth. Finally, Fig. 5 (f) shows the configuration used for the additional target area, with four corner reflectors in formation and a metal wire fence.

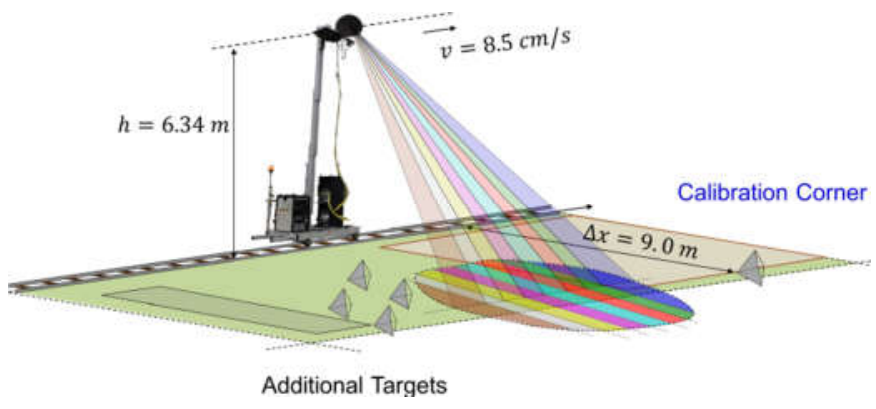

(a)

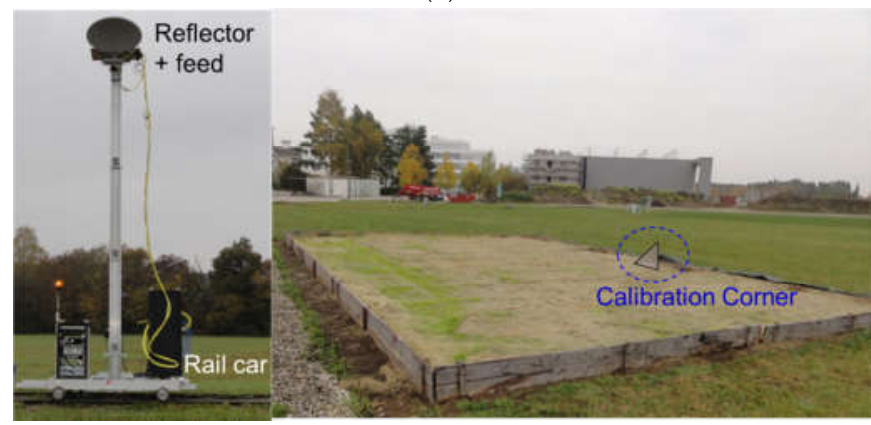

(b)

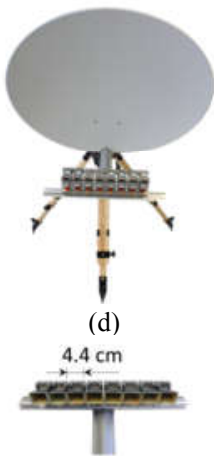

(e)

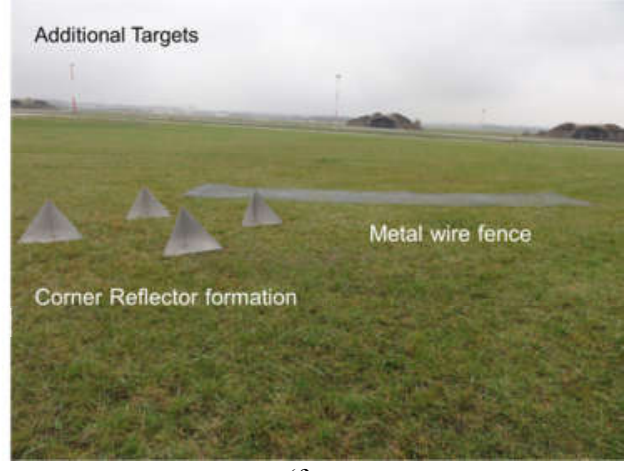

(f)
Fig. 5: Illustration of experimental setup. (a) Schematic representation of the experiment. The radar system is mounted atop a mast of $6.34 \mathrm{~m}$ height on a rail car, which also carries the radar's electronics. The system has 8 channels in azimuth that illuminate different Doppler regions. The scene consists basically of a calibration corner on the sandbox - a target which is present for antenna pattern determination and an additional target area. (b) Close-up of the rail car and the radar system. (c) The calibration corner mounted at the edge of the sandbox. (d) Rx reflector antenna and its feed. (e) Close-up of the feed, which consists of 8 horn antennas of $4.4 \mathrm{~cm}$. (f) Additional target area with a formation of 4 corner reflectors and a metal wire fence.

As seen in TABLE III, the data were acquired at the relatively high sampling rate of $10.0 \mathrm{~Hz}$ (the Doppler bandwidth for each feed channel is around $1.0 \mathrm{~Hz}-\mathrm{cf}$. Fig. 7 (c)) and at uniform sampling. This is intended to allow, in a first step, performing a characterization of the antenna patterns from the calibration corner's response, as detailed in Section IV.B. The pattern information derived from this step is used to compensate phase imbalances between the channels. It is also employed to define the system's array manifold $\left(G_{n}\left(f_{d}\right)\right.$ in (1)), necessary for the second step, the simulation of a multichannel staggered SAR acquisition and reconstruction of the resulting data, described in the next section. 
TABLE III

PROOF OF CONCEPT PARAMETERS

Staggered PRI Sequence Parameters

(used for interpolation/reconstruction)

\begin{tabular}{lll}
\hline Quantity & Symbol & Value \\
\hline Average PRF & $\overline{P R F}$ & $1.25 \mathrm{~Hz}$ \\
Initial PRI & $P R I_{0}$ & $0.891 \mathrm{~s}$ \\
PRI sequence step & $\Delta$ & $-5.31 \mathrm{~ms}$ \\
Sequence length & $N_{P R I}$ & 33 \\
\hline \hline
\end{tabular}

Grid, Reconstruction and Processing Parameters

\begin{tabular}{|c|c|c|}
\hline Quantity & Symbol & Value \\
\hline Output sampling rate & $P R F$ & $10.0 \mathrm{~Hz}$ \\
\hline $\begin{array}{l}\text { Number of samples in azimuth } \\
\text { beamformer window }\end{array}$ & $N_{\text {win }}$ & 56 \\
\hline SNR emphasis parameter & $\alpha$ & $0.1 / 0.5$ \\
\hline $\begin{array}{l}\text { Maximum phase center shift from } \\
\text { input to output grid }\end{array}$ & $\Delta x_{\max }$ & $3.63 \mathrm{~cm}$ \\
\hline Processed Doppler bandwidth & $B W_{\text {proc }}$ & $3.4 \mathrm{~Hz}$ \\
\hline
\end{tabular}

\section{B. Signal Processing and Calibration}

The signal processing of the data can be summarized into four basic steps:

- Pre-processing: conversion of the data of each of the channels to complex format and range compression. The preprocessed data are still not azimuth compressed and are used as input to the antenna pattern analysis.

- Azimuth Antenna Pattern Analysis: the phase and amplitude of the calibration corner's range compressed response is used to estimate the antenna pattern of each of the feed elements.

- Azimuth Calibration: phase-correction of the data, using the output of the antenna pattern analysis. The data after calibration are ready to be azimuth-compressed or interpolated to simulate the staggered acquisition.

- Simulation of a Staggered SAR Acquisition: the calibrated data (uniformly sampled at a high rate) are interpolated and sampled to match a non-uniform grid that corresponds to the staggered PRI sequence in TABLE III (mean sampling rate of $1.25 \mathrm{~Hz}$ ). Afterwards, all 8 channels are used as input to the beamforming, so that the original $10.0 \mathrm{~Hz}$ sampling is restored. The recovered data are then compared to the sum over the azimuth channels (which has the pattern $G_{\text {sum }}\left(f_{d}\right)$ of (5)) of the original data, sampled at $10.0 \mathrm{~Hz}$.

The processing chain, starting from the raw data, is detailed in Fig. 6. The pre-processing of the channels (real data sampled at 1 GS/s) starts with down-conversion from the intermediate frequency $f_{I F}=205 \mathrm{MHz}$ to baseband and conversion to complex I/Q format. Next, the data are range compressed and a correction of the range delays induced by different paths (e.g. different cable lengths and microwave junctions) is performed (cf. [34] for more details on system calibration). A basic radiometric correction is then performed by compensating the range to the second power curve at each range bin.

Next, the azimuth antenna pattern analysis by means of the calibration corner's response is performed. The range compressed data of the corner reflector are used to determine the complex antenna patterns. The amplitude information is used to calculate the manifold for beamforming, whereas the phase information is used to form a correction that compensates the phase differences between the channels, which is the goal of the azimuth calibration step. This follows the assumption that ideally no phase differences are expected between the feed elements (cf. [22]). This stage yields the calibrated data, still not azimuth compressed and in the original uniform grid.

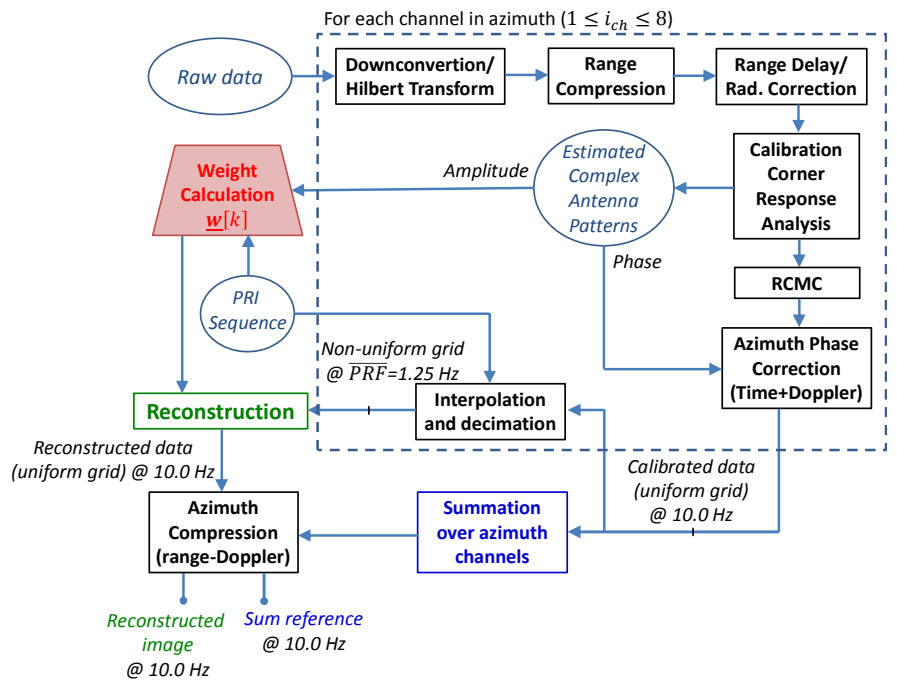

Fig. 6: Block diagram detailing the raw data processing chain. The processing done on each azimuth channel independently is represented inside the dashed line box, and afterwards the various channels are combined for beamforming.

The calibration corner response analysis and the resulting databased calibration procedure are detailed in Fig. 7. The plot in Fig. 7 (a) shows the azimuth profiles (maximum over range, for each position of the platform) for the feed channels. These are color coded consistently with Fig. 5 (a) (e.g. the fore channel in brown and the aft in blue), as throughout the other plots in this paper. The first half of the image contains the corner formation, whereas the second half has the calibration corner as main feature.

Fig. 7 (b) shows the range cell migration of the calibration corner in each of the channels (color-coded plots), compared to the ideal trajectory (dashed black line), i.e.

$R\left(t_{a z}\right)=\sqrt{\left(v_{\text {plat }} \cdot t_{a z}-x_{\text {tgt }}\right)^{2}+r_{0}^{2}}$,

where $x_{t g t}$ is the target's position in along-track. The platform velocity $v_{\text {plat }}$ and the corner's slant range position ${ }^{10} r_{0}$ are listed in TABLE II. A good match is observed, and moreover the effect of the different azimuth antenna patterns is clearly visible. Note that each feed element views the target at a given Doppler region with limited overlap between neighboring feed elements.

\footnotetext{
${ }^{10}$ The value is estimated from the data, as the actual position of the phase center is expected to be over the reflector's surface [39].
} 


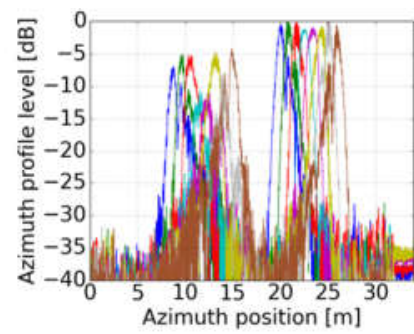

(a)

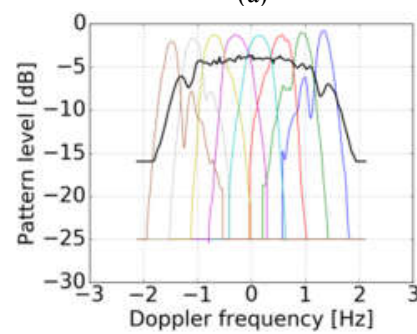

(c)

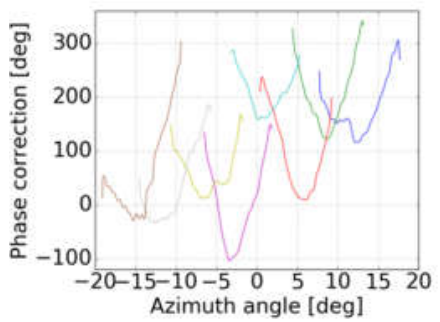

(e)

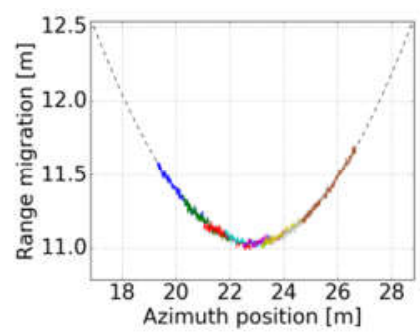

(b)

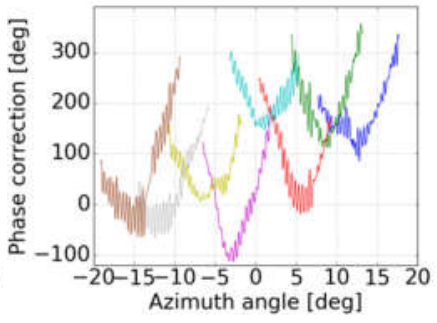

(d)

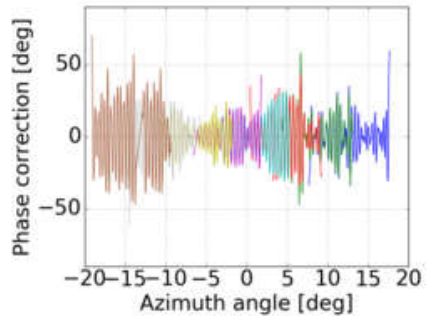

(f)
Fig. 7: Data-based calibration plots, color-coded as in Fig. 5 (a). (a) Profiles over azimuth position - at the maxima over range - of the feed channels. (b) Range cell migration (RCM) of the calibration corner's maxima over range, showing the feed elements individually and a reference RCM in the nominal position of the target (black dashed line). (c) Magnitude of the patterns derived from the calibration corner's response, with resampling to an azimuth Doppler frequency grid (cf. (15))), after low-pass filtering. The black line corresponds to the sum of all feed elements $G_{\text {sum }}\left(f_{d}\right)$. (d) Azimuth phase correction derived from the phase expected from the geometry. (e) Low pass component of the phase correction (effect of the antenna patterns). (f) Remaining high-pass component, attributed to platform motion, namely the vibration of the mast holding the reflector antenna.

Fig. 7 (c) shows the gain of the antenna patterns derived from the profiles. Interpolation of the response and low-pass filtering were applied to remove artifacts. The curve is resampled to an instantaneous Doppler frequency grid, which relates to the slow (along track) time $t_{a z}$ according to [1]

$f_{d}\left(t_{a z}\right)=\frac{2 \cdot v_{\text {plat }}}{\lambda} \cdot \frac{v_{\text {plat }} \cdot t_{a z}-x_{t g t}}{R\left(t_{a z}\right)}$,

where $\lambda$ is the wavelength.

The black line shows the sum $G_{\text {sum }}\left(f_{d}\right)$ of the patterns, assuming no phase difference between them. It has a combined bandwidth of circa $3 \mathrm{~Hz}$. Each feed channel is independently normalized to its maximum after range compression. This causes the peaks of all patterns to be similar, even though the outermost elements are expected to show lower gains w.r.t. the central ones, due to defocusing.
Fig. 7 (d) shows the unwrapped phase difference between the maximum of the corner's response (over range) at each azimuth position and a reference phase. The latter is obtained from an ideal point-target response calculated from the geometry (the range corresponding to (14)), mapped to an azimuth angle grid. This phase has two noteworthy components. First, the phase imposed by the uncompensated azimuth antenna pattern of the $k^{\text {th }}$ feed element $\phi_{\text {pattern }}\left(k, \theta_{a z}\right)$, where $\theta_{a z}$ is the azimuth angle. Second, a phase arising from non-compensated crosstrack motion of the platform $\Delta R_{\text {plat }}\left(t_{a z}\right)$. One may write for the $k^{\text {th }}$ feed element

$\phi_{k}\left(t_{a z}\right)=\phi_{\text {pattern }}\left(k, \theta_{a z}\left(t_{a z}\right)\right)+4 \cdot \frac{\pi}{\lambda} \cdot \Delta R\left(t_{a z}\right)$.

These contributions are separated using the following approach. The low-pass component ${ }^{11}$ seen in Fig. 7 (d) is attributed to the antenna patterns ${ }^{12}$, whereas the remaining high-pass component in Fig. 7 (e) is attributed to platform motion. In fact, closer analysis reveals that the phase oscillation in Fig. 7 (e) occurs in phase between feed channels and is hence feed element independent, as expected. Moreover, the peak amplitude corresponds to circa 2.0 $\mathrm{mm}$. Spectral analysis of the oscillations by means of a power spectral density estimation shows a main component at a frequency of around $1.0 \mathrm{~Hz}$. As a reference, the fundamental frequency of a simple pendulum of length $L$ is given by

$f_{0} \cong \frac{1}{2 \cdot \pi} \cdot \sqrt{\frac{g}{L}}$,

where $g$ is the acceleration due to Earth gravity. For a pendulum with $L=h_{\text {ant }}=6.34 \mathrm{~m}$ (corresponding to the nominal mast length), $f_{0}=1.2 \mathrm{~Hz}$. The results are thus considered to be consistent with small periodical oscillations of the mast holding the reflector during the motion of the rail car. These are due to, e.g., step motor vibration. The small amplitude is moreover consistent with the nearly wind-free meteorological conditions during the data take.

As depicted in Fig. 6, after range cell migration (RCMC), the phase calibration of the feed channel's data is performed. It comprises two steps in different domains, to reflect the nature of the two main error sources mentioned above. The correction of the time-dependent motion contribution (high pass component in Fig. 7 (e), an estimate of the term proportional to $\Delta R\left(t_{a z}\right)$ in (16)) is done in time domain. The azimuth-angle dependent antenna contribution (low pass component in Fig. 7 (d), which estimates $\phi_{\text {pattern }}\left(k, \theta_{a z}\left(t_{a z}\right)\right)$, is done in frequency domain. This explores the relation between Doppler-frequency and squint, and allows performing the correction for all targets simultaneously. In the end of the process, the calibrated data

\footnotetext{
${ }^{11}$ A rectangular low-pass filter was applied with an empirically determined cut-off frequency of $0.6 \mathrm{~Hz}$ to separate the two components in this case.

${ }^{12}$ In [31], an anechoic chamber measurement of the individual feed element's antenna patterns in amplitude and phase is documented. The patterns measured at that time do not directly apply to the current setup, since the system hardware was dismounted and rearranged. Nonetheless, the results shown there bear great resemblance to Fig. 6. In the latter, the maxima of each channel are however equalized by a normalization step after range compression of the individual channels.
} 
show the amplitude patterns in Fig. 7 (c) and nearly no phase distortions between the feed elements. This corresponds to the nominal behavior of a reflector system.

These data are used as input to the simulation of a staggered SAR acquisition. The calibrated data, sampled at $10.0 \mathrm{~Hz}$, is zeropadded in frequency domain to an even higher sampling rate (factor 128) and then linear interpolation in time domain is applied to simulate a staggered PRI acquisition at the sampling instants of the PRI sequence described in TABLE III (a periodically non-uniform grid). In this geometry, staggering of the PRI to avoid blind ranges is not necessary; therefore, a scaled version of a sequence derived for a spaceborne geometry is used $^{13}$. The 33 PRI sequence is scaled here to a mean PRF of 1.25 Hz. After reconstruction (using the beamforming algorithm in Section II) with the 8 channels, the initial sampling of $10.0 \mathrm{~Hz}$ is restored and directly compared to the original data. As indicated in Fig. 6, this step is done individually for each channel.

The calibrated data are also used to create a reference dataset by summing up over the channels. These data are regularly sampled at $10.0 \mathrm{~Hz}$ with an azimuth antenna pattern described by $G_{\text {sum }}\left(f_{d}\right)$ and are referred to as the sum reference (cf. Fig. 6). This is a meaningful reference since it represents the ideal output of the reconstruction. The reconstruction process is the beamforming from Section II. The results are addressed in detail in Section IV.C. As seen in Fig. 6, comparison takes place after azimuth compression with a classic range-Doppler processor [1].

\section{Results}

The reconstruction of the regular grid data sampled at $N_{c h} \cdot \overline{P R F}=10.0 \mathrm{~Hz}$ requires the combination of all channels over a window of several pulses (cf. Fig. 3). Parameters of the input and output grids, as well as the reconstruction algorithm's parameters (cf. Section II) are summarized in TABLE III.

The weights are calculated using the pattern information seen in Fig. 7 (c) as $G_{n}\left(f_{d}\right), 1 \leq n \leq N_{c h}$. The phase center positions, are obtained from the PRI sequence parameters. The black curve in the same figure is $G_{\text {sum }}\left(f_{d}\right)$, adopted as $G_{\text {common }}\left(f_{d}\right)$. We choose $N_{\text {win }}=56$, but consider two scenarios for the choice of the sensitivity parameter $\alpha$, namely 0.1 and 0.5 . The goal is to compare the results with a relatively low and a relatively high SNR emphasis, to better illustrate the trade-offs involved. The results are summarized in Fig. 8. Fig. 8 (a) shows the impulse responses for the two reconstructed images ( $\alpha=0.1$ and $\alpha=0.5$ ) and the sum reference. The impulse responses are stable around the main target position but show differences in the sidelobe region, both with respect to the reference and to each other. It should be pointed out that the effect of resampling errors due to the imperfect goal pattern implementation (cf. [22] for a more detailed discussion) is twofold: On the one hand, the residual "non-regularity" due to phase center position errors (meaning the output grid is not exactly uniform) leads to residual ambiguities. Their levels (which can be estimated from the

\footnotetext{
${ }^{13}$ The example is chosen from a HRWS system design in [21], [22]. There, the design procedure of

[11] is applied to cover a $350 \mathrm{~km}$ swath with $3 \mathrm{~m}$ resolution at a mean PRF of $2700 \mathrm{~Hz}$.
}

difference to the reference) are, however, seen to be small. On the other hand, the deviations of each output sample's pattern from $G_{\text {sum }}\left(f_{d}\right)$ mean that also the average antenna pattern is not exactly that of the reference. This is not a cause of aliasing per se, but it means that the reconstructed data have a different spectral weighting then the reference. This results in a change of the shape of the impulse response as a whole. Fig. 8 (b) is a zoom of the main beam showing that this effect is small. All profiles achieve a $3 \mathrm{~dB}$ resolution of $2.5 \mathrm{~cm}$.

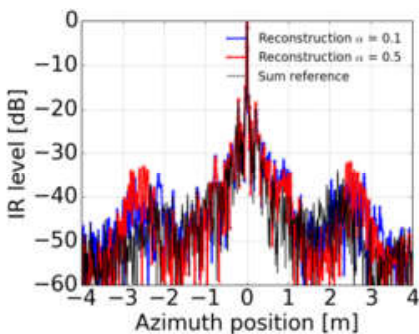

(a)

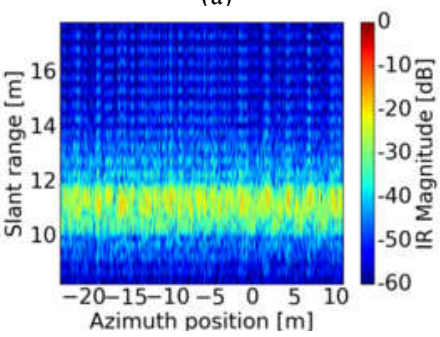

(c)

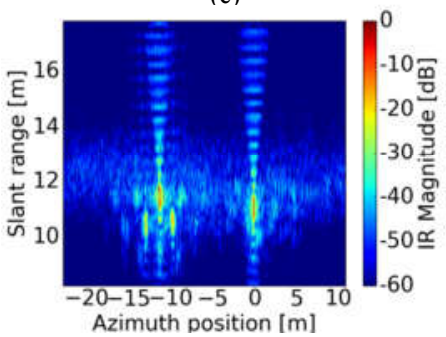

(e)

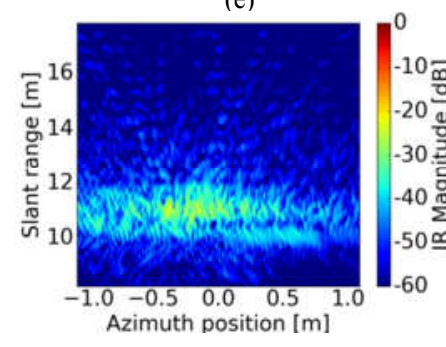

(g)

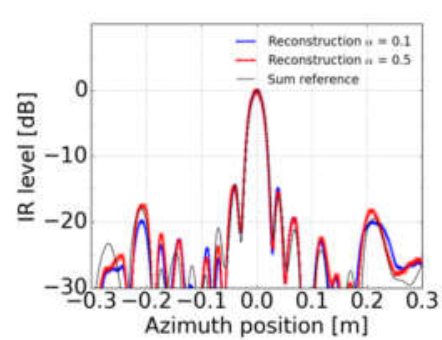

(b)

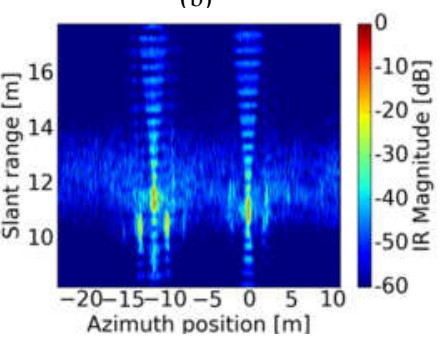

(d)

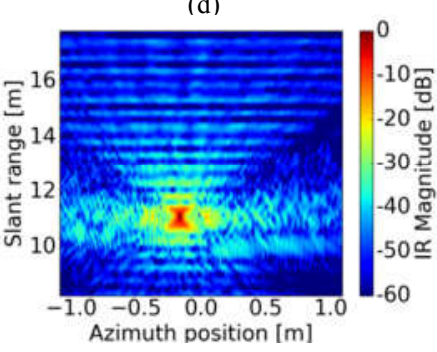

(f)

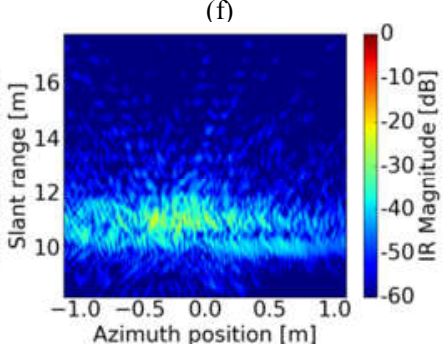

(h)

Fig. 8: Images after reconstruction and azimuth compression. (a) Calibration corner's azimuth profile after compression, comparing the result of the two sets of reconstruction weights with the sum reference. (b) Zoom of the same impulse responses to highlight the fine $2.5 \mathrm{~cm}$ resolution achieved. (c) Result of simply azimuth compressing the non-uniformly sampled data (low quality reference). (d) Image of the sum reference channel at the uniform grid with $10.0 \mathrm{~Hz}$ sampling (upper quality bound). (e) Reconstructed image obtained with the low SNR emphasis set of weights ( $\alpha=0.1$ ) (f) Zoom around the calibration corner reflector for the high SNR emphasis set of weights $(\alpha=0.5)$. $(\mathrm{g})$ Magnitude squared difference between the reconstructed image with $\alpha=0.1$ and the sum reference. (h) Magnitude squared difference to the reference for the case $\alpha=0.5$. 
Fig. 8 (c) shows the result of azimuth compressing the sum over the feed channels of the non-uniformly sampled data. The result is highly ambiguous. The approach is equivalent to ignoring the non-uniformity and is not a meaningful processing strategy. It is, nonetheless, intended as a lower quality bound, to highlight the importance of the resampling. Fig. 8 (d) in turn shows the upper quality bound, i.e. the sum reference of Fig. 6(corresponding to perfect reconstruction). The azimuth position axis has its origin at the position of the calibration corner.

The corner constellation is visible in the azimuth region $[-15,-10] \mathrm{m}$. The narrow elevation pattern is also clearly observable. It is a consequence of the usage of a single element of the feed array in elevation, and causes the attenuation of the response of the corner in near range. Fig. 8 (e) is the reconstructed image with $\alpha=0.1$, showing in general great similarity to the reference. The effect of the sidelobe distortions is visible as "clutter", in the region with magnitude below -30 dB. Fig. 8 (f) shows a zoom around the calibration corner's response for the image reconstructed with $\alpha=0.5$. As was the case in Fig. 8 (a), no major image artifacts are observed.

To better illustrate the residual reconstruction errors, Fig. 8 (g) and Fig. 8 (h) show the squared magnitude of the difference between the reconstructed and reference images, respectively for $\alpha=0.1$ and $\alpha=0.5$. The shown area is again a zoom around the calibration corner. The sidelobe distortions are visible at peaks below $-20 \mathrm{~dB}$ and in most regions the error level lies below $-25 \mathrm{~dB}$, a level expected due to the limited accuracy of the radar system setup and calibration. All images were normalized to their respective maximum magnitude.

Reconstruction with both $\alpha=0.1$ and $\alpha=0.5$ showed similar results, which is interesting as an indication that the increased SNR emphasis does not severely degrade the ambiguity suppression. The main difference between the two sets of weights and motivation for the mixed form of the costfunction (6) is however the achieved SNR scaling $\Phi_{S N R}$ (cf. (12)). In the case of $\alpha=0.1, \Phi_{S N R}=-3.9 \mathrm{~dB}$; whereas for $\alpha=0.5, \Phi_{S N R}=-0.9 \mathrm{~dB}$. In the latter case, the very high SNR of the data did not allow this considerable difference of the achieved SNR scaling to be observed. Therefore, to better illustrate the noise behavior of the processing strategies, a final case study is explained, employing the addition of synthetic white noise.

As indicated in Fig. 9 (a), noise was added to the interpolated irregularly sampled data before the azimuth beamforming and compression (compare to block diagram in Fig. 6). The level is intended to establish a noise floor of around $-30 \mathrm{~dB}$. The noisy data are reconstructed with the two sets of weights, leading to two different reconstructed images and the achieved noise levels are analyzed in the further plots of Fig. 9. All the analysis is done before performing the azimuth compression, in order to emphasize the impact on the output patterns, without masking due to posterior filtering steps. Fig. 9 (b) shows the profile of the calibration corner of the first reconstructed image, i.e., with the low SNR emphasis set of weights $(\alpha=0.1)$, against instantaneous Doppler.

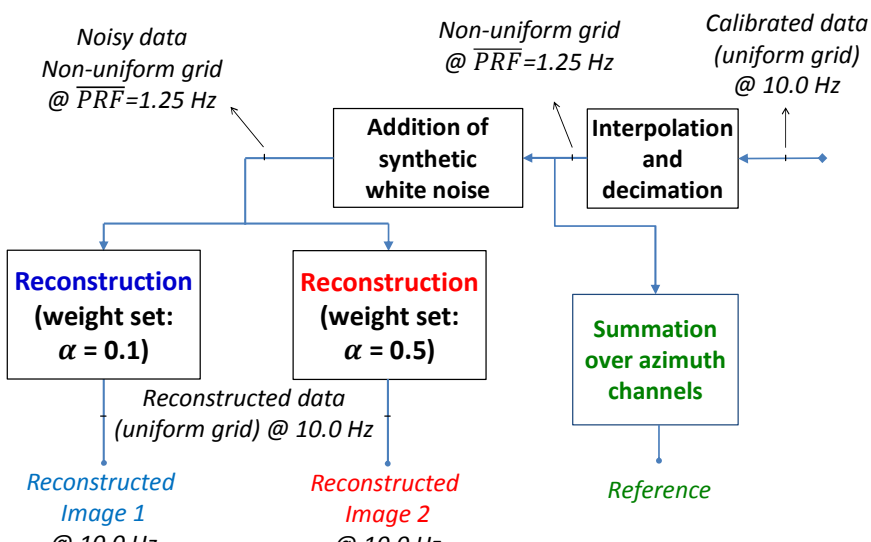

(a)

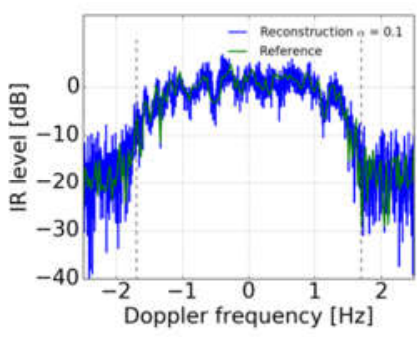

(b)

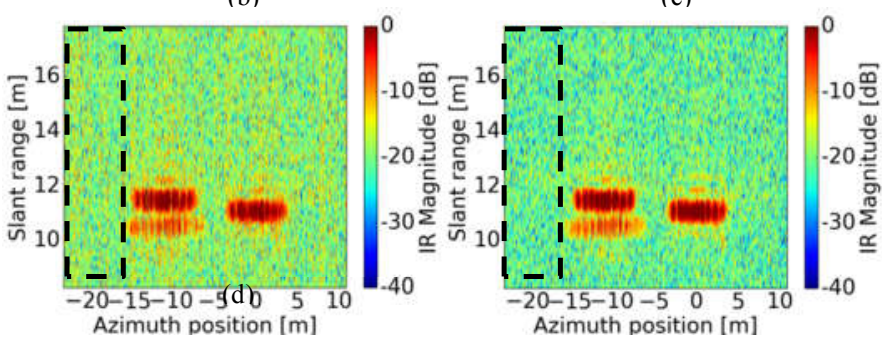

Fig. 9: Analysis of noise scaling properties of reconstruction. (a) Block diagram for the performed analysis, indicating the addition of synthetic white noise before the reconstruction to compare the scaling of the two set of weights, leading to two reconstructed images. The reference formed by summing the channels in the irregular grid is provided as a visual aid for the profiles to be shown. (b) Profile of the calibration corner after reconstruction for the low SNR emphasis set of weights ( $\alpha=0.1$, corresponding to the first reconstructed image) plotted against instantaneous Doppler, resembling the sum pattern. (c) Profile of the calibration corner after reconstruction for the higher SNR emphasis set of weights ( $\alpha=0.5$, corresponding to the second reconstructed image). (d) First reconstructed image, before azimuth compression, including an indication of the "noise-only" area used for variance estimation to access noise levels (black dashed line box). (e) Second reconstructed image, also before azimuth compression.

The reference shown is derived from the non-uniformly sampled data by summing over the channels and intended as a visual aid. Both profiles are normalized to their respective mean levels within the processed bandwidth (highlighted by the vertical dashed lines) and resemble the sum pattern $G_{\text {sum }}\left(f_{d}\right)$, as expected. Fig. 9 (c) shows the same profile for the high SNR emphasis set of weights $(\alpha=0.5)$, and the reference is repeated. The second profile is visibly less contaminated by noise. Fig. 9 (d) shows the first reconstructed image, and the responses of the 
four corner formation (left of plot), as well as the calibration corner (around the origin of the axis) are visible.

The box (dashed black line) indicates a region which contains mostly noise. It is used for a variance estimation to validate the noise scaling prediction. Fig. 9 (e) shows the second reconstructed image. It is seen to show a lower noise floor, due to the higher SNR emphasis of the weights. To quantify the improvement, the variance of the images in the region highlighted in Fig. 9 (d),(e) was estimated and compared. The ratio of the variances between Fig. 9 (d) and Fig. 9 (e) was found to be $3.2 \mathrm{~dB}$, which validates the predicted difference in $\Phi_{S N R}$.

\section{Part II: Planar Antenna Systems}

\section{MultichanNel StagGered SAR With PlANAR DIRECT-RADIATING ARRAYS}

The primary beam steering technique in [36] clearly requires the usage of a reflector antenna. Interestingly, even though motivated by the former one, the azimuth resampling strategy of Section II does not. Knowledge of the array manifold vector is assumed, but no special structure is imposed on it, meaning the application can be readily extended to non-reflector antennas. In particular, planar direct-radiating antenna systems, so far the default in radar satellite technology, represent a natural candidate. Successful application of the technique, however, requires proper choice of the goal patterns. In fact, the optimum approximation is not necessarily a good one, if the desired patterns are poorly chosen, in the sense of not being realizable. In this context, an analysis of the phase center resampling capabilities of planar systems is desirable to provide a better understanding of how realizable a set of patterns of the form in (3) is. The system is assumed to be a phased array (common antenna for $\mathrm{Tx}$ and $\mathrm{Rx}$ ) with multiple independently digitized channels on receive in both elevation and azimuth. The multiple elevation elements allow the implementation of SCORE with multiple elevation beams [15],[16]. Multiple azimuth elements are required to allow the application of the resampling strategy of Section II.

A fundamental difference which affects the manifold extension discussed in Section II is the increased influence of the geometry for planar arrays. In the case of reflectors, all physical channels (described by the patterns $G_{n}\left(f_{d}\right)$ in (1) have the same phase center, whereas for planar arrays each channel shows a distinct phase center position. This means that in (1) the phase ramp defining the input sample position is also influenced by the array's geometry. Temporal and spatial sampling are thus combined. In contrast, for reflector systems, the relative position between samples is determined exclusively by $t_{R X}[i]$. This is in fact exploited as part of the VBS method formulation (cf. Section II) by using different pulses as input to the beamformer $\left(N_{p}>1\right)$ in order to provide a phase center diversity that the receive channels alone do not possess in the reflector case. In contrast, the planar antenna system's channels already show a variety of phase center positions, corresponding to the position of each element. Therefore, adding additional pulses to the extended manifold has a smaller impact with respect to the resampling, even though the extension remains possible and to some extent desirable.
As shown in Appendix B, a given phase center position $x_{P H}$ within the array is only achievable if the weights given to the elements are Hermitian-symmetric with respect to that point. Since the array is finite and sampled at the element positions, this narrows down the possible phase centers considerably. Namely, if an odd number of elements is taken (including the trivial case of one element), the possible phase centers coincide with the element positions. If an even number of elements is taken instead, they coincide with the positions in-between adjacent elements. In any case, the achievable phase centers are the geometrical centers of the array and of any sub-arrays contained therein. Any other positions cannot be exactly obtained over the whole set of angles.

Moreover, the regularization of the samples requires not only that several phase centers - forming the regular grid - be obtained $\left(t_{\text {out }}[k]\right.$ phase ramp component in (3)). The patterns are furthermore required to remain stable enough across the grid, for the output to be equivalent to a single channel $\left(G_{c o m}\left(f_{d}\right)\right.$ component in (3)). In other words, it is not enough to obtain the different phase centers if these are subject to different amplitude modulations (denoted $B\left(f_{s p}\right)$ in Appendix B) for each position in the output grid.

Note that, in the reflector case, the resampling algorithm is able to modify the phase centers continuously within certain bounds, while maintaining stable amplitude. This is achieved by steering of the primary beams. In contrast, in the planar case, the choice of the output phase centers is achieved by directly activating portions of the azimuth aperture. Therefore, planar systems show an inherent discretization of the achievable phase centers, and the same can be said of the available amplitude modulations, which are not stable over combinations with a different number of elements. This means that this class of systems is subject to limitations with respect to the capability of positioning the phase centers under the stable amplitude constraint.

To achieve the desired pattern stability, a fixed number of elements should be activated. This can be achieved by designing the azimuth antenna so that the combination of an integer number of neighboring elements yields the aperture size required by the resolution and then setting $G_{\text {com }}\left(f_{d}\right)=G_{\text {sub-set }}\left(f_{d}\right)$, the pattern of this sub-set of the antenna array elements. This will lead the algorithm to always combine this fixed number of elements, but to choose the combination with a geometric center (determined in this case by both array geometry and pulse sampling) closest to the desired phase center $\left(t_{\text {out }}[k]\right.$ in (3)). The length of the active aperture is thus set, but the maximum extent of the phase center shift remains a function of the total antenna size. The granularity or "resolution" of the phase center shift is in turn determined by the inter-element spacing (alongside the PRF, when $N_{p}>1$ and several pulses are used). As before, the mean PRF still defines the maximum gap size and thus the maximum required phase center shift.

Clearly, a better performance is expected for higher PRFs and a finer division of the aperture through a larger number of elements, but the more complex interdependency between geometry and PRI sequence should be considered in the design. A joint design of the antenna (in particular the element positions) and the PRI 
sequence $^{14}$ is key to limit the required shifts and ensure good resampling performance. A possible approach is to fix the antenna size and choose the lowest possible mean PRI and number of elements which lead the shifts to be small enough to achieve the required performance level.

Regarding the choice of beamforming parameters, the approach of combining a smaller number of pulses than in the reflector case is favored. The reason is that the manifold extension bears the possible disadvantage of reducing the signal's SNR (cf. [22]). Moreover, even though some level of SNR emphasis $(\alpha>0$ in (6),(9)) can still be applied, the solution that maximizes the SNR becomes namely the addition of all array elements $G_{\text {sum }}\left(f_{d}\right)$ [25]. It shows a very distinct amplitude modulation (cf. (31)) in comparison to e.g. the single-element pattern, and cannot be realized in phase center positions deviating strongly from the geometrical center of the array. In conclusion, a limited success of the SNR-MSE compromise is expected in this case. Some SNR gain is nevertheless possible with moderately low levels of $\alpha$, leaving the control of the baseline decorrelation effect in the extended manifold to the aforementioned restriction in the number of pulses $N_{p}$ used as input.

The main differences in the design guidelines with respect to the reflector case can be summarized as:

- The common pattern $G_{c o m}\left(f_{d}\right)$ should be the pattern $G_{\text {sub-set }}\left(f_{d}\right)$ of the combination of a sub-set of the antenna array elements. A single element is the simplest case, but in general a combination of several neighboring elements is necessary. $G_{\text {sub-set }}\left(f_{d}\right)$ must achieve the Doppler bandwidth required by the resolution and defines the length of the active aperture.

- The phase center deviations required by the resampling should be minimized by means of the joint design of the azimuth antenna and the PRI sequence. The total antenna size and the number of azimuth channels should be chosen under the constraint that the combination of an integer number of neighboring elements yields the active aperture length. For instance, the total antenna length can be fixed and then $N_{c h}$ set to the smallest number of channels which yields appropriate performance, using for each geometrical configuration a favorable mean PRI.

- The number of pulses $N_{p}$ in the input sample window should be reduced (thus limiting $N_{\text {win }}=N_{p} \cdot N_{c h}$ and improving SNR performance) and a small emphasis $\alpha \cong 0$ set, to improve ambiguity suppression.

This design approach will be illustrated next in Section VI.

\section{Simulation Results For Planar ANTENNA}

This section illustrates the design approach described in Section V with an example of a single-pol multichannel staggered SAR mode. The aim is a $1.5 \mathrm{~m}$ azimuth resolution over a $400 \mathrm{~km}$ swath, still enough to provide a global coverage in 7 days. As in Section III, AASR, RASR and NESZ levels are required to be better than $-25 \mathrm{~dB}$.

\footnotetext{
14 The fast PRI variation criterion is still applied in this case. The joint design means in this context changing the sequence design input parameters in conjunction with the antenna characteristics.
}

The parameters for the planar system are summarized in TABLE IV. As pointed out in Section V, to assure an adequate performance, the design of the azimuth antenna and the PRI sequence must be done simultaneously. In this case, to achieve the resolution of $1.5 \mathrm{~m}$, an antenna of length $3.0 \mathrm{~m}$ in azimuth is considered as the sub-set which needs to be activated for each channel, thus determining $G_{c o m}\left(f_{d}\right)$. The total antenna length was chosen to be $15.0 \mathrm{~m}$, due to gain considerations.

TABLE IV

Planar Single-Pol SimUlation Scenario Parameters

\begin{tabular}{lll}
\hline \multicolumn{3}{c}{ Platform and Swath Parameters } \\
\hline Quantity & Symbol & Value \\
\hline Orbit height & $h_{\text {orbit }}$ & $700 \mathrm{~km}$ \\
Swath width on ground & $W_{\text {ground }}$ & $400 \mathrm{~km}$ \\
Swath minimum/maximum & $\phi_{\text {min }} / \phi_{\max }$ & $22.0^{\circ} / 42.8^{\circ}$ \\
(off-nadir) look angle & \\
\hline \hline
\end{tabular}

\begin{tabular}{lll}
\hline \hline \multicolumn{3}{c}{ Antenna Parameters } \\
\hline Quantity & Symbol & Value \\
\hline Antenna height in elevation & $h_{e l}$ & $6.0 \mathrm{~m}$ \\
Antenna length in azimuth & $l_{a z}$ & $15.0 \mathrm{~m}$ \\
Center frequency & $f_{0}$ & $1.2575 \mathrm{GHz}$ \\
Number of channels in & $N_{e l} / N_{a z}$ & $36 / 15$ \\
elevation/azimuth & $d_{e l} / d_{a z}$ & $0.7 \lambda / 1.0 \mathrm{~m}$ \\
Channel spacing in elevation/azimuth & $\phi_{t i l t}$ & $32.6^{\circ}$ \\
Elevation tilt angle & $L_{\Omega}$ & $2.0 \mathrm{~dB}$ \\
Antenna system losses &
\end{tabular}

\begin{tabular}{lll}
\hline \multicolumn{2}{c}{ Pulse and Tx/Rx Hardware Parameters } \\
\hline Quantity & Symbol & Value \\
\hline Average PRF (both polarizations) & $\overline{P R F}$ & $2050 \mathrm{~Hz}$ \\
Initial PRI & $P R I_{0}$ & $520 \mu \mathrm{s}$ \\
PRI sequence step (between pulses of & $\Delta$ & $-2.7 \mu \mathrm{s}$ \\
same polarization) & $N_{P R I}$ & 25 \\
PRI sequence length & $T_{d c}$ & $6 \%$ \\
Pulse duty cycle & $B w_{c h i r p}$ & $85 \mathrm{MHz}$ \\
Pulse (chirp) bandwidth & $P_{T X}$ & $32.0 \mathrm{~W}$ \\
Peak transmit power of a Transmit- & $\bar{P}_{T X}$ & $1040.0 \mathrm{~W}$ \\
Receive Module & $T_{\text {noise }}$ & $649 \mathrm{~K}$ \\
Average transmit power & - & $\mathrm{V}$ \\
System noise temperature & \\
Transmitted polarizations &
\end{tabular}

\begin{tabular}{|c|c|c|c|}
\hline \multicolumn{4}{|c|}{ Processing Parameters } \\
\hline \multicolumn{2}{|l|}{ Quantity } & Symbol & Value \\
\hline \multicolumn{2}{|c|}{ Goal azimuth resolution } & $\delta_{a z}$ & $1.5 \mathrm{~m}$ \\
\hline \multicolumn{2}{|c|}{ Processed bandwidth } & Bw $w_{\text {proc }}$ & $5343 \mathrm{~Hz}$ \\
\hline \multicolumn{2}{|c|}{$\begin{array}{l}\text { Number of simultaneous elevation } \\
\text { beams }\end{array}$} & $N_{\text {beams }}$ & 4 \\
\hline $\begin{array}{l}\text { Elevation } \\
\text { beamforming }\end{array}$ & $\begin{array}{l}\text { Within swath } \\
\phi \in\left[\phi_{\min }, \phi_{\max }\right]\end{array}$ & \multirow[b]{2}{*}{$20 \cdot \log _{10}\left(\epsilon_{S D L}\right)$} & $-33 \mathrm{~dB}$ \\
\hline $\begin{array}{l}\text { sidelobe } \\
\text { constraints (look } \\
\text { angle dependent, } \\
\text { cf. Fig. 15) }\end{array}$ & $\begin{array}{l}\text { Near-normal } \\
\text { incidence }\left(\phi \leq 6^{\circ}\right)\end{array}$ & & $-45 \mathrm{~dB}$ \\
\hline \multicolumn{2}{|c|}{$\begin{array}{l}\text { Number of samples in azimuth } \\
\text { beamformer window }\end{array}$} & $N_{\text {win }}$ & 45 \\
\hline \multicolumn{2}{|c|}{ SNR emphasis parameter } & $\alpha$ & 0.0 \\
\hline \multicolumn{2}{|c|}{$\begin{array}{l}\text { Hamming window coefficient (over } \\
\text { azimuth) }\end{array}$} & $\phi_{\text {hamm }}$ & 0.9 \\
\hline
\end{tabular}


The simplest case would then be the juxtaposition of five $3 \mathrm{~m}$ long panels, each of which corresponding to a channel. A channel spacing of $3.0 \mathrm{~m}$ leads however to relatively high phase center shifts, as illustrated in Fig. 10 (a), in which the minimum, mean and maximum (over the swath positions, which have different gaps due to pulse blockage) is portrayed as a function of the mean PRF.

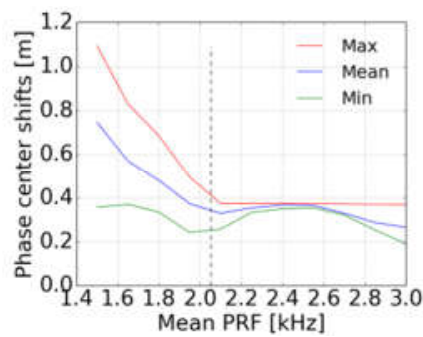

(a)

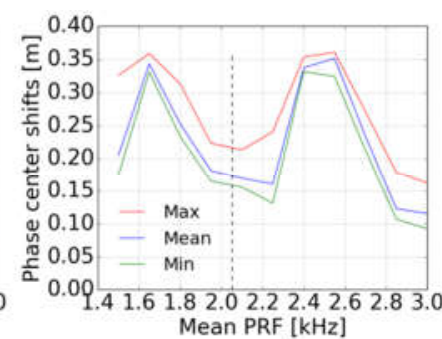

(b)
Fig. 10: Statistics over the swath's ranges for the required phase center shifts as function of the mean PRF. The total antenna length is $l_{a z}=15.0 \mathrm{~m}$. In (a) the antenna consists of 5 elements of 3.0 meter length, whereas in (b) 15 elements of $1.0 \mathrm{~m}$ length are considered. (In this case the same effective aperture can be achieved by activating 3 elements). The mean PRF of $2050 \mathrm{~Hz}$ (vertical dashed line) is chosen to minimize the required phase center shifts with respect to the original sampling.

In order to reduce these shifts while keeping a reasonably low mean PRF, the desired active aperture was split into 3 elements of $1.0 \mathrm{~m}$. Given the antenna length of $15 \mathrm{~m}$, this results in a 15 channel system. Its maximum shifts are seen in Fig. 10 (b). Note that the active aperture is kept the same ( $3.0 \mathrm{~m}$ long), by keeping the same common pattern as design goal and, as a consequence, the algorithm will tend to activate 3 elements at a time, taken from the 15 available channels at each pulse position. An overlap between the activated apertures is made possible by the subdivision into $1.0 \mathrm{~m}$ elements. The effect is a finer sampling in terms of the original channel's phase centers. It thus allows a reduction of the maximum shift for comparable mean PRFs, in comparison to the previous case. The adopted mean PRF of 2050 $\mathrm{Hz}$ is indicated as a vertical black dashed line and is seen to lead to small shifts while being reasonably low.

The beamforming in elevation consists of the use of a phaseonly pattern [37] on transmit and SCORE on receive. The elevation beamforming technique of Appendix A is used to simultaneously control the position of the maximum (following the echo on ground) and the sidelobes (limited to $33 \mathrm{~dB}$ below the main signal, within the swath) and thus improve the range ambiguity rejection. Especially low sidelobe levels are imposed at near-normal incidence ( $-45 \mathrm{~dB}$ w.r.t. main signal), to compensate for the increased reflectivity at nadir (cf. A.IV).

The beamforming in azimuth also consists of phase-only patterns on transmit - in order to increase the beamwidth without sacrificing the transmitted power - whereas relying on the method of Section II on receive. Following the remarks on Section V, no SNR emphasis was used $(\alpha=0.0)$, but the extended manifold was restricted to a relatively narrow vicinity of 3 pulses, meaning the total of samples is
$N_{\text {win }}=3 \cdot 15=45$. The narrow window indirectly enhances the SNR, compensating the lack of SNR emphasis. The key performance results are provided in Fig. 11. The azimuth resolution goal of $1.5 \mathrm{~m}$ is achieved. The AASR is better than $-27.1 \mathrm{~dB}$ in the swath extending from 285 to $685 \mathrm{~km}$ ground range. In this case, the effect of the nadir returns over the RASR is seen to be appreciable, as shown by the peaks in the red curve, including the power of nadir returns, in comparison to the blue one, in which they are not included. This is due to the higher level of the planar array's pattern at nadir in comparison to the (more directive) reflector antenna. The additional suppression achieved by elevation beamforming (cf. A.IV) is important to achieve the RASR peak level of $-25.5 \mathrm{~dB}$, satisfying the requirement. In this scenario, the smearing of the nadir returns due to the PRI staggering leads to an attenuation of circa $15 \mathrm{~dB}$. This effect could, if necessary, be increased by the use of longer, compound sequences (cf. more elaborated sequences in [11]). The NESZ is better than $-25.0 \mathrm{~dB}$. An important effect of the nadir return suppression achieved by elevation beamforming is seen in the worst-case NRSR plot. It is kept in this case close to $0 \mathrm{~dB}$, meaning that the nadir returns are close to the noise floor. Should no suppression be applied, these returns would be visible in the SAR image.

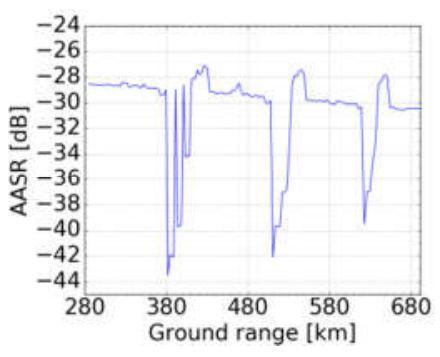

(a)

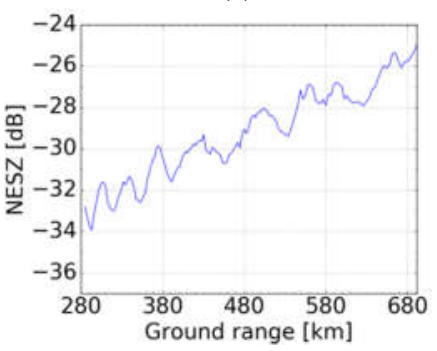

(c)

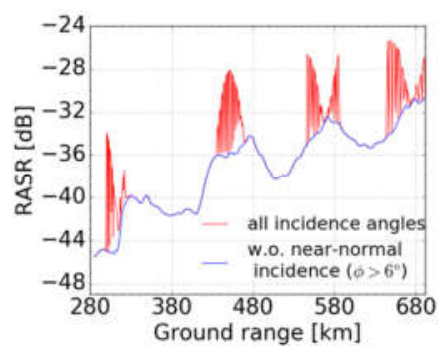

(b)

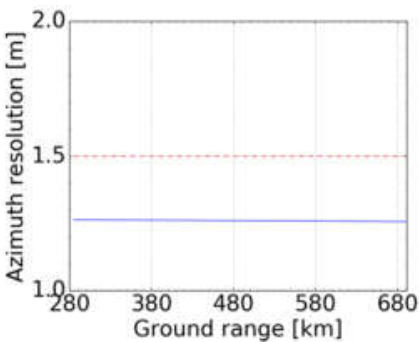

(d)

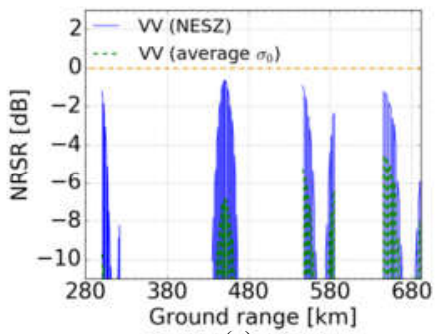

(e)

Fig. 11: Key performance parameters over swath for single-pol mode of planar system: (a) AASR, (b) RASR with (red) and without (blue) the contribution of nadir returns, (c) NESZ, (d) azimuth resolution and (e) NRSR both with respect to the NESZ (noise floor) and the average $\sigma_{0}$, significant in this case. 
The implementation of an antenna of $15.0 \mathrm{~m}$ length in L-band is recognized to be challenging. A similar design in e.g. C-band, however, could prove a more readily implementable option, after certain adaptations. The antenna length is indeed comparable to that already in use in ESA's Sentinel-1 [38] and the Canadian Space Agency's Radarsat [39] C-band radar missions.

\section{REVIEW AND FINAL REMARKS}

This paper reviewed and experimentally demonstrated the multichannel staggered SAR resampling technique described in [22]. Moreover, additional aspects of the method when applied to system with planar direct radiating array antenna were discussed. This architecture is currently the default technology for spaceborne SAR.

A reflector antenna based design in single polarization was analyzed. It is meant as a complement to [22], in which a quadpol system is considered. The system images a $500 \mathrm{~km}$ swath with $1.0 \mathrm{~m}$ azimuth resolution, exceeding the performance of all state-of-the-art SAR systems by more than one order of magnitude. The predicted performance of an example of system design with a planar antenna architecture was also provided. It illustrates the approach's potential with a swath of $400 \mathrm{~km}$ at $1.5 \mathrm{~m}$ azimuth resolution. In general, the analysis suggests that the new multichannel staggered SAR mode and the proposed Virtual Beam Synthesis (VBS) technique enable a new class of high-resolution ultra-wide-swath SAR systems with unprecedented imaging performance. The new concepts and techniques proposed in this paper can be applied to both reflector and planar antenna architectures operating in single, dual or quad polarization mode.

Comparing the reflector and the planar antenna systems [40] in multichannel staggered SAR operation, it was shown that the latter present some limitations with respect to the phase center resampling capabilities of the former. For reflectors the output phase center can be varied continuously by beamforming of the primary feed elements. The major limitation is imposed by the reflector size, which sets the maximum possible shift. This translates into a lower bound on the mean PRF, with clear implications for the maximum swath width. In contrast, for planar arrays the phase center positions are discrete and the interchannel spacing is decisive to minimize the shifts. The minimization should be done in conjunction with a proper design of the PRI sequence. Even though the antenna length remains a relevant parameter, the performance is in practice mostly limited by the maximum number of azimuth channels. This important parameter scales the cost and complexity of the system design.

Both systems can, however, yield similar performance levels. This requires the design of the planar array to be adapted to take the different physical properties in consideration and enough azimuth channels. More channels than in the reflector case tend to be required. Planar systems have the advantage of representing a more mature technology in the context of SAR satellites. Reflector systems (particularly unfurlable mesh reflector antennas) are on the other hand an important trend for new spaceborne SAR missions [41]. They possess many desirable features positively influencing overall SAR performance, not specifically linked to multichannel modes. These include better sidelobe levels (leading to e.g. better range ambiguity suppression) and the potential to deploy larger apertures in space and thus improve the antenna gain.

The paper also included a first proof of concept of the processing strategy, employing data acquired with a reflector and a multichannel feed in X-band. The comparison between the reconstructed images and the reference sampled at a regular grid shows successful recovery by the newly developed Virtual Beam Synthesis technique. Furthermore, the noise scaling behavior of the beamforming was validated using synthetic white noise, due to the high SNR of the original data.

\section{APPENDIX A: ELEVATION BEAMFORMING}

The main novelty and difficulty of the multichannel staggered SAR modes involves the sampling configuration in the azimuth dimension. Correspondently, azimuth beamforming is the solution of the resampling problem. Nonetheless, elevation beamforming remains a topic of interest for such systems. It represents an important ancillary technique to improve the overall performance in terms of range ambiguities and signal-to-noise ratio, taking into account particularities of the imaging mode's operation. This appendix thus shifts focus from the azimuth to the elevation dimension. Section A.I introduces a new elevation beamforming concept which is especially suited for staggered PRI systems. Its application is however not necessarily restricted to them. In Section A.II, the concept is illustrated and the resulting beams are compared to the outputs of other currently in use beamforming alternatives (cf. [8]). An example reflector antenna with a multichannel feed array is analyzed. Section A.III discusses the implications of the elevation beamforming for SAR performance in terms of RASR.

\section{A.I - The Sidelobe-constrained Beamformer}

As discussed in [8] in the context of reflector systems, beamforming techniques relying on knowledge of the patterns are essential to implement the SCan On REceive (SCORE) [14] beamforming concept. The rationale of this technique is to follow the echoes of interest over elevation. The objective is to suppress range ambiguities and to improve the overall gain. The $\mathrm{Rx}$ gain compensates the low gain of the broad Tx pattern that illuminates a wide swath in elevation. As discussed in Section I, several simultaneous SCORE elevation beams are assumed, mapping subswaths. The operation is inherently time-dependent, as a change of the position of the maximum of the beam over elevation with time is required.

To define the notation, assume the channel's elevation beams to be described by $G_{n}(\phi), 1 \leq n \leq N_{e l}$, where $\phi$ is the off-nadir look angle ${ }^{15}$ described in Fig. 12. The antenna system is thus described by an array manifold vector [25]

$$
\underline{\boldsymbol{G}}_{e l}(\phi)=\left[\begin{array}{lll}
G_{1}(\phi) & \ldots & G_{N_{e l}}(\phi)
\end{array}\right]^{T},
$$

\footnotetext{
${ }^{15}$ One may equivalently write $G_{n}\left(\phi-\phi_{\text {tilt }}\right)$, but the more concise notation with respect to $\phi$ is preferred to emphasize the SCORE operation principle.
} 
which collects the $N_{e l}$ complex antenna patterns. Let the $N_{e l}{ }^{-}$ dimensional complex elevation beamforming weight vector be denoted by $\boldsymbol{w}$ and the resulting pattern of the array at a particular elevation angle by $G_{\text {array }}(\phi)=\underline{\boldsymbol{w}}^{H} \cdot \underline{\boldsymbol{G}}_{\boldsymbol{e l}}(\phi)$. Moreover, allow the statistics of the noise affecting the system's channels to be described by the $N_{e l}$ by $N_{e l}$ noise covariance ${ }^{16}$ matrix $\underline{\boldsymbol{R}}_{\text {noise }}$.

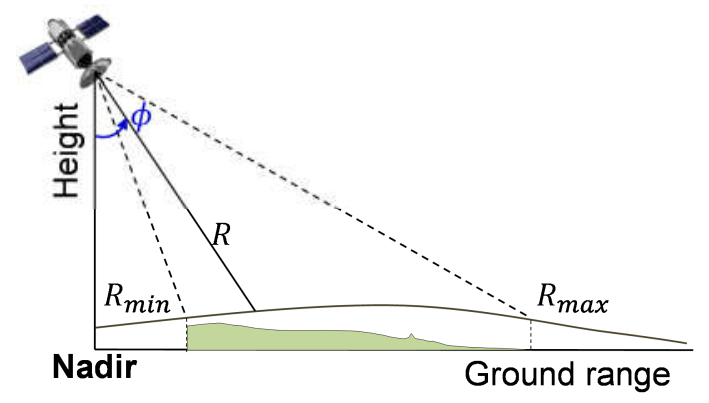

Fig. 12: Swath geometry for elevation beamforming. The off-nadir look angle is denoted $\phi$. The antenna main axis is pointed at $\phi_{t i l t}$, so that the elevation angle in the antenna's coordinate system is $\phi-\phi_{\text {tilt }}$.

A beamformer of particular interest in the implementation of SCORE beams is the Minimum Variance Distortionless Response (MVDR) [8],[25],[44]. The MVDR is the solution to the constrained optimization problem:

$\underline{\boldsymbol{w}}_{\text {MVDR }}=\begin{gathered}\operatorname{argmin}\left(\underline{\boldsymbol{w}}^{H} \cdot \underline{\boldsymbol{R}}_{\text {noise }} \cdot \underline{\boldsymbol{w}}\right) \\ \text { subject to: } \underline{\boldsymbol{w}}^{H} \cdot \underline{\boldsymbol{v}}\left(\phi_{0}\right)=1\end{gathered}$.

The name MVDR is because the cost function being minimized $\sigma_{\text {noise }}^{2}=\underline{\boldsymbol{w}}^{H} \cdot \underline{\boldsymbol{R}}_{\text {noise }} \cdot \underline{\boldsymbol{w}}$ represents the variance or power of the noise in the output after the weighting. The constraint $\underline{\boldsymbol{w}}^{H} \cdot \underline{\boldsymbol{v}}\left(\phi_{0}\right)=1$ means the signal is not distorted at the look angle of interest $\phi_{0}$. This is typically the intended maximum of the SCORE beam in this context. The solution to (19) can be derived using Lagrange multipliers and yields [8],[25], [44]

$\underline{\boldsymbol{w}}_{M V D R}=\frac{\underline{\boldsymbol{R}}_{\text {noise }}^{-1} \cdot \underline{\boldsymbol{v}}\left(\phi_{0}\right)}{\underline{\boldsymbol{v}}\left(\phi_{0}\right)^{H} \cdot \underline{\boldsymbol{R}}_{\text {noise }}^{\mathbf{1}} \cdot \underline{\boldsymbol{v}}\left(\phi_{0}\right)}$.

In the particular case of white noise, $\underline{\boldsymbol{R}}_{\text {noise }}=\boldsymbol{I}$, the identity matrix. The elements are hence weighted by $\underline{\boldsymbol{v}}\left(\phi_{0}\right)^{H} /\left\|\underline{\boldsymbol{v}}\left(\phi_{0}\right)\right\|^{2}$ and the beamformer has a "matchedfilter" behavior, gathering all the available power at $\phi_{0}$. MVDR thus steers the beam towards the echo signal direction of arrival and aims at maximizing the SNR. It does not, however, consider that the pulse has a non-vanishing extension on the ground.

Even though the MVDR is very effective at maximizing the output SNR while focusing the maximum beam's gain around a $\phi_{0}$ of choice, it allows no control over the sidelobes of the achieved beam. Motivated by this, [44] analyses an extension of the optimization problem with addition of a sidelobe constraint. This is done by introducing a maximum level $\epsilon_{S D L}$ for the formed beam at a grid of $N_{S D L}$ elevation angles $\Theta_{S D L}=\bigcup_{n} \phi_{n}, 1 \leq n \leq N_{S D L}$. The resulting problem is:

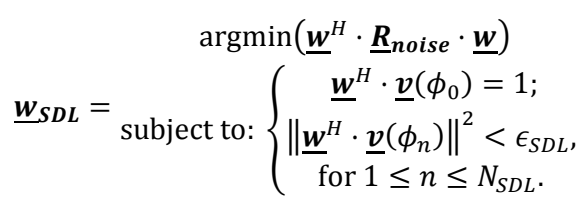

This means that the distortionless constraint is kept, but a maximum level of the sidelobes within $\Theta_{S D L}=\bigcup_{n} \phi_{n}$ is specified. The problem (21) does not have a closed form solution in every case and is not necessarily feasible for every choice of pattern or sidelobe level $\epsilon_{S D L}$. Nonetheless, as demonstrated in[44], it can be re-written as a Second-Order Cone (SOC) Optimization problem which can be efficiently solved by existing optimization packages [45]. Moreover, highlevel interfaces such as [46] allow the problem to be specified directly in the form (21). An additional interesting feature of the SOC solvers is the capability of testing for feasibility before attempting to solve the problem, which allows adaptive strategies for the threshold $\epsilon_{S D L}$, as e.g. relaxing the constraint until a solution is possible.

The technique is applicable both for reflector antennas and for planar direct radiating arrays, as only knowledge of the manifold is assumed. It should be recalled that a global sidelobe level smaller than a given $\epsilon_{S D L}$ in the planar case can be readily achieved by conventional Dolph-Chebyshev weighting [21], with the advantage of a closed-form solution, but the optimization technique allows additional flexibility, since $\Theta_{S D L}$ can be chosen freely. An interesting possibility is setting extended minima at specific angular intervals. Another readily available extension are angle-dependent sidelobe levels $\epsilon_{S D L}\left(\phi_{n}\right)$, which can also be handled seamlessly by the numeric solvers and may be of interest for certain scenarios.

The sidelobe control strategy described above is of special interest in the context of SCORE pattern design, and can be used in order to improve the range ambiguity-to-signal ratio (RASR). This is done by specifying the direction of arrival of the signal as $\phi_{0}$ and including the angles of arrival of the ambiguities (and their vicinity) within $\Theta_{S D L}$.

The rationale is similar to the use of another beamforming technique described in [8] (employed for the same purpose of improving the RASR), namely the Linear Constraint Minimum Variance (LCMV) [25]. The latter beamformer solves the problem

$\underline{\boldsymbol{w}}_{L C M V}=\begin{gathered}\operatorname{argmin}\left(\underline{\boldsymbol{w}}^{H} \cdot \underline{\boldsymbol{R}}_{\text {noise }} \cdot \underline{\boldsymbol{w}}\right) \\ \text { subject to: } \underline{\boldsymbol{w}}^{H} \cdot \underline{\boldsymbol{A}}=\underline{\boldsymbol{c}}\end{gathered}$,

where the $N_{e l} \times N_{e l}$ array matrix

$\underline{\boldsymbol{A}}=\left[\underline{\underline{v}}\left(\phi_{0}\right) \quad \underline{\boldsymbol{v}}\left(\phi_{1}\right) \quad \ldots \quad \underline{v}\left(\phi_{N_{e l}-1}\right)\right]$

\footnotetext{
${ }^{16}$ In practice it is often unknown, and thus estimated from the data.
} 
contains the manifold for the angle of interest $\phi_{0}$ and $N_{e l}-1$ other angles for which the pattern level can be specified. Typically, in the case of SCORE beamforming,

$\underline{\boldsymbol{c}}=\left[\begin{array}{llll}1 & \epsilon_{S D L} & \ldots & \epsilon_{S D L}\end{array}\right]$

and $\epsilon_{S D L}$ is chosen to be zero or a very low value. This creates a grid of nulls or minima $\Theta_{S D L}=\bigcup_{n} \phi_{n}, 1 \leq n \leq N_{e l}-1$. The analogy between (22)-(24) and (21) is thus clear, replacing the "hard" equality constraints by "soft" inequality constraints over the sidelobe grid $\Theta_{S D L}$ and adjusting the size of this grid.

The LCMV beamformer has indeed the advantage of a closed form solution [8]

$\underline{w}_{L C M V}=\underline{R}_{\text {noise }}^{-1} \cdot \underline{A} \cdot\left(\underline{A}^{H} \cdot \underline{R}_{\text {noise }}^{-1} \cdot \underline{A}\right)^{-1} \cdot \underline{c}^{T}$,

but the number of constraints is limited by the number of available elevation channels $N_{e l}$.

In the context of staggered SAR, the position of the range ambiguities changes from pulse to pulse [27]. This means that typically several tenths or even hundreds of different angles of arrival need to be considered for each order of ambiguity. The LCMV is thus of limited usefulness in this situation, though very effective for a conventional constant-PRI SAR. In contrast, the sidelobe-constrained beamformer of (21) can be used to force a minimum (though not a zero) over a broad region (not necessarily contiguous), as no a-priori restriction on $N_{S D L}$ exists. In fact, $N_{S D L}$ should ideally be high, since a properly fine grid is necessary to ensure that the pattern behavior follows the constraints in the vicinity of the grid points. This allows the creation of "broad minima", again making an LCMV-like approach feasible for mitigation of range ambiguities in staggered SAR.

\section{A.II - Elevation Beamforming Method Comparison}

This section presents example SCORE elevation beams to illustrate the application of the discussed methods and compare their properties. Patterns from the reflector-antenna system described in TABLE I are employed. To simplify the discussion, a single azimuth channel is considered (namely the central one out of the nine), and the elevation patterns are taken at an azimuth angle of $0.0^{\circ}$. The combination of the channels over azimuth and the implications for the range ambiguity performance are considered at a later stage, in Section A.III.

The goal is to implement the SCORE beams at far range. In this region, the range ambiguities are closer to the main beam (in terms of the corresponding elevation angles) and pattern defocusing effects contribute to higher sidelobes. These effects tend to degrade range ambiguity performance. As will be shown in the following example, this effect may be mitigated by the elevation beamforming. A look angle $\phi_{0}=43.2^{\circ}$, at the edge of the swath of interest, is considered the desired maximum of the beam (note that the antenna is tilted $32.5^{\circ}$ with respect to nadir). Besides, a total of $N_{e l}=9$ elevation channels are chosen as part of the input manifold for beamforming, as seen in Fig. 13 (a). The plot refers to the individual feed element's directivity, as output of GRASP.

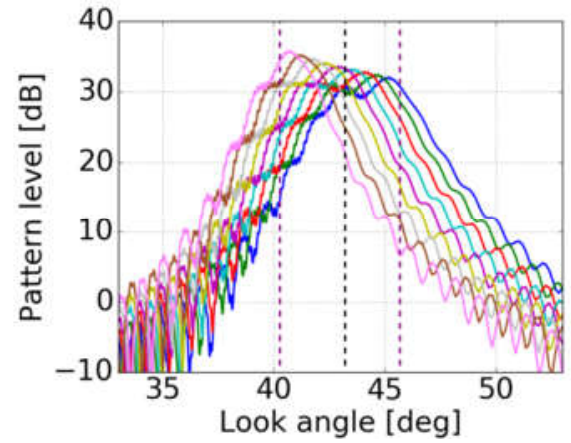

(a)

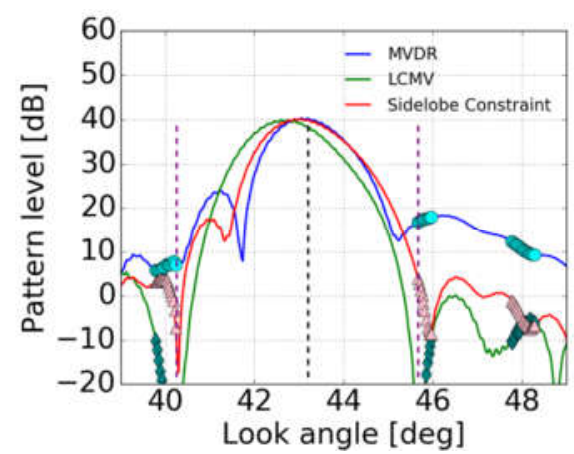

(b)

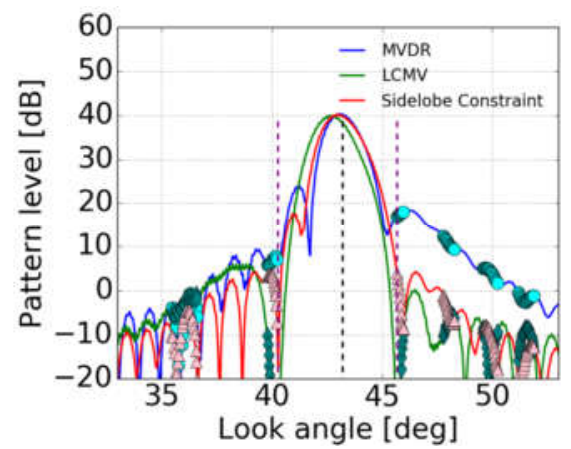

(c)

Fig. 13: Far-range SCORE elevation beams illustrating the discussed methods of elevation beamforming. (a) Manifold over elevation consisting of the 9 active elements used to form the beams. The beam maximum - by design set at the direction of arrival of the signal - is indicated by the central vertical dashed line and the beginning of the sidelobe region (derived from the geometry and the PRI sequence parameters in TABLE I) by the side vertical dashed lines. (b) SCORE beams formed by the different methods (MVDR, LCMV and Sidelobeconstrained beamformers respectively in blue, green and red). The markings for signal direction and sidelobe region are repeated, and the location of the range ambiguities is superimposed on the different patterns (circles, diamonds and triangles, respectively); (c) SCORE patterns of (b) over a broader range of angles to better illustrate the behavior of the outermost range ambiguities.

In the plot, the desired position of the maximum is highlighted by the vertical black dashed line in the center. The limits of the sidelobe region - where the range ambiguous contributions begin - is marked by purple vertical dashed lines to its sides. 
The position of the ambiguities is determined by the geometry and the PRI sequence parameters. The noise affecting the channels is assumed white, so $\underline{\boldsymbol{R}}_{\text {noise }}=\boldsymbol{I}$, the identity matrix.

Fig. 13 (b) and (c) show the patterns (the gain as defined by [25]) obtained by the algorithms described in the previous section. The first shows a zoom around the main beam. The second depicts a broader angular region.

The MVDR beam (cf. (20)) is seen in blue, and the levels in the positions of the range ambiguities are highlighted by light blue circles. Note that several positions exist $\left(N_{P R I}=28\right.$ per order $)$ due to the staggered PRI operation. This solution effectively maximizes the gain at the goal elevation angle $\phi_{0}$, but provides no control over the sidelobes. The first ambiguity to the right of the main beam is seen to be at a relatively high level, circa $-20 \mathrm{~dB}$ below the pattern's maximum. The LCMV beam (cf. (25)) is calculated by setting a linear constraint (cf. (24)) of 1.0 at $\phi_{0}$ and $\epsilon_{S D L}=0$ for eight range-ambiguous positions. The pattern is seen in green, and ambiguous positions are highlighted by adjacent green diamonds.

Whereas only the aforementioned eight ambiguities can be nulled, the neighboring ones are also seen to be suppressed by the proximity of the deep minima formed (especially visible in Fig. 13 (b)). However, no control is possible over more distant ones. The same is true regarding the position of the main beam, which is seen to be distorted, showing a maximum at a look angle circa $0.5^{\circ}$ smaller than $\phi_{0}$. Finally, the sidelobeconstrained pattern (cf. (21)) is seen in red. It is obtained with $10 \cdot \log _{10} \epsilon_{S D L}^{2}=-36.0 \mathrm{~dB}$ and $\Theta_{S D L}$ corresponding to all angles of the sidelobe grid (i.e. the expected range ambiguity positions) within $\left[15.3^{\circ}, 40.3^{\circ}\right]$ and $\left[45.7^{\circ}, 48.2^{\circ}\right]$. The grid spacing is $0.03^{\circ}$. This time, ambiguities are highlighted by triangles and their maximum is at circa $4 \mathrm{~dB}$. As the maximum gain is at $40 \mathrm{~dB}$, this indicates a successful implementation of the threshold.

The robustness of the beamformers against errors in the coefficients is also a crucial point for their applicability in realworld systems, where effects such as limited phase accuracy play a role. A first assessment and comparison of the methods with this regard is performed by means of a Monte Carlo simulation. This consists of the following steps. First, the nominal weights are calculated from the error-free manifolds according to the specific algorithm in Section A.I. Then, each element of the complex weight vectors is disturbed with a uniform phase draw from a uniform distribution in the interval $\left[-\xi_{u} / 2, \xi_{u} / 2\right]$. For a varying $\xi_{u}$ in the interval $[0,45.0]^{\circ}$, a total of 100 draws of the phases are done. For each of them, the disturbed patterns are calculated. For consistency, the same disturbance (drawn phase errors) is used for the three beamforming methods at each trial. For each drawn phase, pattern parameters are assessed. The mean over the trials is finally considered as the expected value.

In Fig. 14 (a) the expected sidelobe-to-peak ratios of the patterns are analyzed for the three beamforming algorithms, shown in different colors. Solid lines indicate the maximum over the sidelobe regions (i.e. range ambiguities), whereas the dashed lines indicate the mean over these range-ambiguous positions. For the LCMV, the maximum over the desired nulls is also provided as a dotted line. It is clear that the MVDR shows very stable levels, nearly invariant to the phase errors, even though the sidelobe-to-peak levels (which translate into RASR levels) are the highest. Both the LCMV and the sidelobe-constrained beamformer show some sensitivity to errors. The nulling of the patterns does not hold in the presence of errors and the maximum of the controlled ambiguities quickly rises (as indicated by the dotted line). However, the maximum level of the sidelobe region is dominated by the non-controlled ambiguities until $\xi_{u} \cong 15^{\circ}$. This means that the performance is not severely degraded for small phase errors. Though the sensitivity (inclination of the curve in $\mathrm{dB} /{ }^{\circ}$ ) increases for larger errors, the peak level remains the lowest of all methods. The sidelobeconstrained beamformer in turn shows a higher starting point (at the set threshold of $-36.0 \mathrm{~dB}$ ) and a smaller sensitivity (which is influenced by the starting point as well as by the weights themselves). The mean curve indicates that for larger phase errors, the sidelobes are on average lower for the latter method in comparison to LCMV, even though the peak values of both methods converge in the extreme case of $\xi_{u} \cong 45^{\circ}$. Both methods are seen to outperform the MVDR beamformer in terms of sidelobe levels, even in the presence of errors.

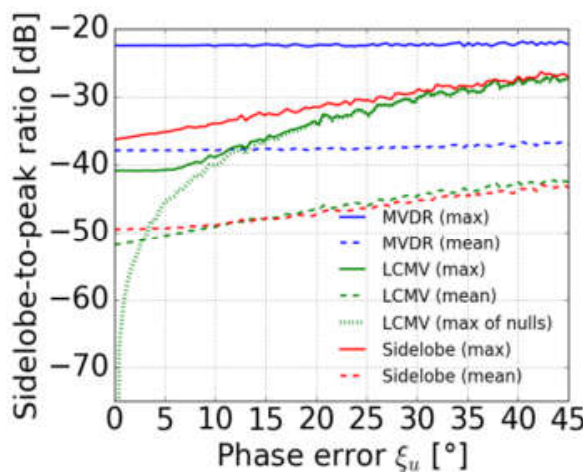

(a)

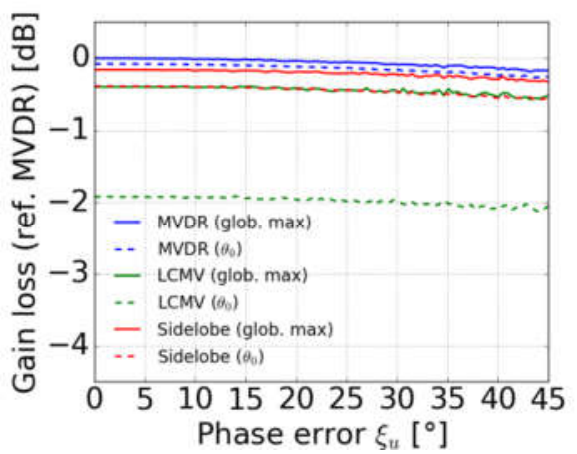

(b)

Fig. 14: Analysis of the impact of weight errors for the different beamforming algorithms. (a) Monte Carlo simulation of sidelobe-to-peak ratio (sidelobes are defined by the sidelobe grid) of the implemented patterns as a function of a uniform phase error in the interval $\left[-\xi_{u} / 2, \xi_{u} / 2\right]$. The MVDR, LCMV and Sidelobe-constrained beamformers are represented by blue, green and red lines, respectively, and for each beamformer the maximum and mean over the sidelobe grid are represented by solid and dashed lines, respectively; (b) Monte Carlo simulation of the gain loss (with respect to the maximum gain of the error-free MVDR), as before as a function of the uniform phase error parameter $\xi_{u}$. 
The influence of the errors over the pattern gain is analyzed in Fig. 14 (b). The metric is the gain with respect to the best case, namely that of the error-free MVDR. The plot shows the gain loss - negative values indicate attenuation - of the global maximum of the patterns (not necessarily at $\phi_{0}$ ) in solid lines. The gain loss at $\phi_{0}$ is shown in dashed lines. The beamformer algorithms are represented by different colors. All methods show relatively small gain sensitivity to the phase errors, with the LCMV method showing the maximum error-induced variation of $-0.2 \mathrm{~dB}$. The error-free levels thus dictate the performance. The MVDR shows the highest gains and a very small discrepancy between the maximum and the gain at position $\theta_{0}$, in this case due to discretization of the elevation grid of the patterns $\left(\phi_{0}\right.$ is not necessarily a sample in the grid). In contrast, the LCMV shows considerable main beam distortion (as visible in Fig. 13 (b) and (c)), with an attenuation of $2 \mathrm{~dB}$ at $\phi_{0}$. It mainly characterizes mispointing, as the absolute maximum is only $0.4 \mathrm{~dB}$ below the MVDR reference. The sidelobe-constrained beamformer shows an intermediate behavior. The attenuation at $\phi_{0}$ is $0.4 \mathrm{~dB}$ and mispointing is mild, with a global maximum showing $0.16 \mathrm{~dB}$ attenuation.

The comparison shows that, as expected, the sidelobeconstrained beamformer provides a compromise between the pattern's gain and sidelobe levels. Its characteristics are inbetween those of the MVDR and the LCMV methods. The gain reduction is a consequence of the additional constraint in (21) in comparison to (19). The achieved minimum noise variance indeed cannot be smaller than in the MVDR case. It should be stressed that the choice of $\epsilon_{S D L}$ and $\Theta_{S D L}$ is very important for meaningful results. A sidelobe region too close to the main beam or a too low $\epsilon_{S D L}$ may severely degrade the achieved gain. With proper design of the parameters, however, the method offers the possibility of avoiding severe main beam distortion (which reduces gain at the signal's direction of arrival) while retaining improved sidelobe suppression.

\section{A.III - Range Performance of Elevation Pattern Comparison Example}

The last section provided a discussion of the elevation beamforming algorithms' properties by means of an example. The implementation of far range SCORE beams of the reflector antenna system described in TABLE I was considered. For simplicity, only the central azimuth channel was analyzed. This section extends that example by considering additional effects arising from the combination of the channels and compares the beamformers in terms of the final SAR system performance. The parameters $\phi_{0}=43.2^{\circ}$ and $N_{e l}=9$ are kept.

Fig. 15 (a) shows, plotted in different colors, the SCORE elevation patterns as function of the look angle, for each of the 9 azimuth elements. Each pattern is taken at the azimuth angle which represents the maximum of the particular channel (recall that for reflector systems the antenna patterns are not separable in azimuth and elevation, and show different maxima positions due to the feed element's displacement). The patterns cannot be clearly distinguished, indicating great similarity between them (apart from the different azimuth angles of the maxima from which the elevation cuts originate).

The signal and ambiguity positions region are highlighted by the green and red crosses. The imposed constraint for the sidelobe is marked by the dashed horizontal line. $\Theta_{S D L}$ in this case includes the look angles in the intervals $\left[15.3^{\circ}, 40.3^{\circ}\right]$ and $\left[45.7^{\circ}, 48.2^{\circ}\right]$. These are chosen to include all orders of range ambiguities, assuming the maximum PRI in the sequence, $439.8 \mu \mathrm{s}$. As in Sections II and VI, the patterns are interpolated to a grid of spacing $0.03^{\circ}$, and the constraint parameter is $10 \cdot \log _{10} \epsilon_{S D L}^{2}=-36.0 \mathrm{~dB}$. Due to the similarity of the patterns from different azimuth elements, the constraint levels are also very similar. The pattern levels below the constraint indicate that the elevation beamforming was successful for all channels.

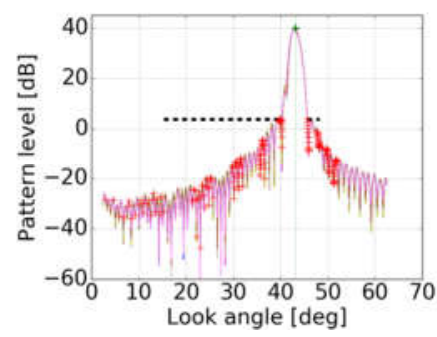

(a)

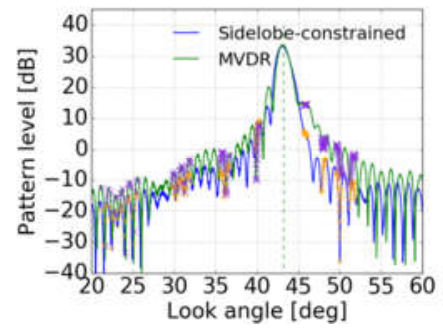

(c)

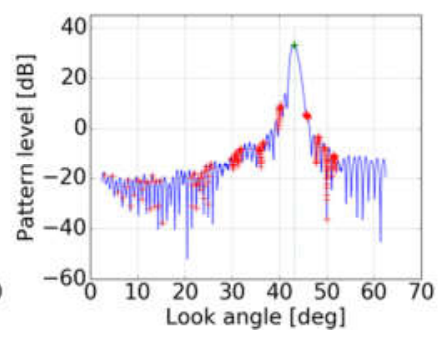

(b)

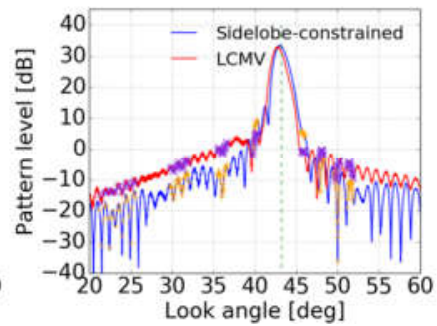

(d)
Fig. 15: Far-range SCORE elevation beams illustrating some special aspects of the elevation beamformers. (a) SCORE elevation patterns (sidelobe-constrained) for each of the azimuth elements at their maximum position. The beam maximum is indicated by a green cross, the contribution from the several ambiguities by red crosses and the imposed constraint for the sidelobe levels - at least $36 \mathrm{~dB}$ below the main peak - by a black horizontal dashed line. (b) Sum over the azimuth elements of the sidelobe-constrained patterns. The cut is taken at an azimuth angle of $0.0^{\circ}$ and the signal and ambiguity position markings are repeated. (c) Comparison of the SCORE patterns of the sum over the azimuth channels (cut around an azimuth angle of $0.0^{\circ}$ ) for the sidelobe-constrained beamformer (blue) and the conventional MVDR (green). The signal direction is highlighted by a vertical dashed line and the range ambiguity positions by ' + ' and ' $\mathrm{X}$ ' symbols superimposed on the respective patterns. The blue patterns are the same as in (b), repeated to facilitate the comparison; (d) Comparison of the SCORE patterns for the sidelobe-constrained beamformer (blue) and LCMV (red) with respective ambiguities.

Fig. 15 (b) shows a pattern which is the result of the summation over the azimuth elements taken at an azimuth angle of $0.0^{\circ}$. It is relevant as the reference common pattern. The signal and ambiguity positions are marked as before. Note that the constraint is no longer fulfilled, since the azimuth angle is no longer that of the maximum for each channel. A considerable 
reduction in sidelobe levels is nevertheless observed, resulting in improved range ambiguity suppression. To better illustrate this point, Fig. 15 (c) shows the SCORE pattern of the central azimuth element in far range (look angle of $43.2^{\circ}$ ) for the sidelobe constrained beamformer (blue) and the conventional MVDR (green). The main beam (centered around the vertical dashed line) is not considerably changed. The sidelobe suppression at the range ambiguity positions (compare the ' + 's and ' $\mathrm{X}$ 's) is however notable, especially for the first-order far range ambiguities. In Fig. 15 (d) the sidelobe-constrained (blue) elevation beam at $0.0^{\circ}$ in azimuth is compared to the LCMV one (red). The latter technique was also applied for each azimuth channel at the location of its maximum. Therefore, due to the non-separable characteristic of the patterns, the deep minima (cf. Fig. 13 (b)) are not visible for the sum of all patterns. Reduced levels are nonetheless obtained.

The impact of the choice of beamformer on system performance in this particular range is summarized in TABLE V. The sidelobe-gain compromise mentioned in Section A.II is seen to be translated into a RASR-NESZ compromise. The sidelobeconstrained beamformer retains the behavior of an intermediate solution between the MVDR and the LCMV with regard to both parameters. As expected, the MVDR technique yields the optimal NESZ of $-26.4 \mathrm{~dB}$ and the LCMV the best ambiguity suppression, with an RASR of $-30.5 \mathrm{~dB}$. The MVDR shows however the worst RASR, with $-23.6 \mathrm{~dB}$, and the LCMV's mispointing with respect to the signal direction leads to a worsened NESZ of $-24.6 \mathrm{~dB}$. The sidelobe-constrained beamformer leads to an NESZ of $-26.0 \mathrm{~dB}$ and an RASR of $-29.6 \mathrm{~dB}$. This indicates that a considerable gain in range ambiguity suppression could be achieved for the price of a slight SNR degradation if compared to the MVDR beamformer.

TABLE V

FAR-RANGE PERFORMANCE OF DIFFERENT ELEVATION BEAMFORMERS

\begin{tabular}{lll}
\hline Beamforming Method & RASR $[\mathrm{dB}]$ & NESZ $[\mathrm{dB}]$ \\
\hline MVDR & -23.6 & -26.4 \\
LCMV & -30.5 & -24.6 \\
Sidelobe-constrained & -29.6 & -26.0 \\
\hline \hline
\end{tabular}

\section{A.IV - DBF for Nadir Return Suppression}

As discussed in [29], the $\sigma_{0}$ (radar cross section per unit area) can be expressed as the sum of two components, $\sigma_{0}=\sigma_{0}^{i n c}+\sigma_{0}^{c o h}$. The coherent (specular) component $\sigma_{0}^{c o h}$ has a Dirac-delta behavior, dominating for nearly normal incidence but quickly vanishing for larger angles. In contrast, the incoherent component $\sigma_{0}^{i n c}$ has lower values but is more stable. In Fig. 16 (a), the two components of the $\sigma_{0}$ are plotted as a function of the incidence angle. Here, the $\sigma_{0}^{i n c}$ curve (from [28]) represents average values, and is used for the RASR estimation. In contrast, $\sigma_{0}^{c o h}$ represents a worst-case scenario, following parameters derived from backscatter measurements in L-band [29]. The Dirac-delta behavior of $\sigma_{0}^{\text {coh }}$ is approximated by an exponential, which reaches $-20 \mathrm{~dB}$ for $0.5^{\circ}$ and has a peak value of $15 \mathrm{~dB}$ (corresponding to a very smooth surface). The backscatter values of the coherent component depend on the topography, soil roughness and moisture, so that a meaningful average curve is difficult to achieve. Since the modelled backscatter is a worst- case rather than the average, the coherent contribution is not included as part of the RASR but rather of a new quantity, the Nadir Return-to-Signal Ratio (NRSR). In order to assess the potential impact on low backscatter areas of the image, this is calculated assuming the signal is at the noise level ( $\left.\sigma_{0}=\mathrm{NESZ}\right)$. The levels with respect to the average backscatter are also included in the plots as a reference.

The implication of the Dirac-delta behavior of $\sigma_{0}^{c o h}$ is that localized spikes in the nadir return power are expected (cf. Fig. 11 (e)). These are seen over the ranges whose range ambiguities (for any PRI, in the staggered SAR case) arise from nadir. The DBF strategy discussed in this appendix can however be used to suppress these returns as well. Fig. 16 (b) shows an example SCORE beam for the system of TABLE IV. The sidelobe level within the swath $\left(\phi \in\left[22.0^{\circ}, 42.8^{\circ}\right]\right)$ is $-33 \mathrm{~dB}$, but it is reduced to $-45 \mathrm{~dB}$ for near-normal incidence $\left(\phi \leq 6^{\circ}\right)$. The low levels cause suppression of nadir returns, ensuring adequate return levels in spite of the increased reflectivity close to nadir. The technique is especially relevant for the less directive planar systems. A further investigation of nadir returns and corresponding compensation strategies, including the influence of calibration errors upon the achievable suppression levels is part of further work on the topic. Data-based relative pattern calibration techniques such as [42] could play an important role in improving system performance with this regard.

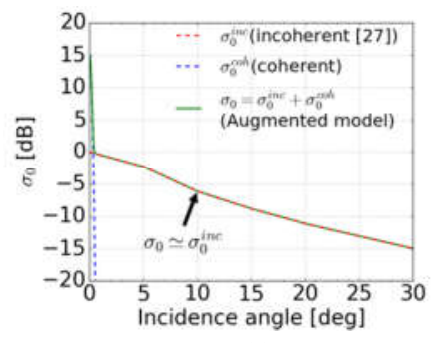

(a)

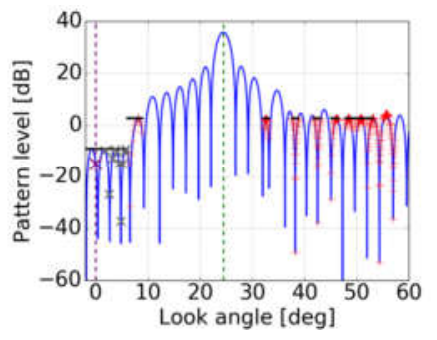

(b)
Fig. 16: Nadir returns and corresponding DBF strategy. (a) $\sigma_{0}$ curve with an incoherent (non-specular) component following [28] and a coherent (specular) deltalike component [29]. The latter is only relevant for nearly normal incidence, and shows a peak value circa $15 \mathrm{~dB}$ above the maximum of the incoherent component. (b) Example Rx elevation pattern for the planar system of TABLE IV at a look angle $\phi=24.5^{\circ}$. The sidelobe constraint (cf. black horizontal lines) within the swath $\left(\phi \in\left[22.0^{\circ}, 42.8^{\circ}\right]\right)$ is $-33 \mathrm{~dB}$, but $12 \mathrm{~dB}$ lower for the nearly normal incidence region $\left(\phi<6^{\circ}\right)$. The ambiguities outside this region are marked by red '+' signs, whereas the ambiguities within the region are marked by ' $x$ ' signs. The main signal and the nadir return are highlighted by vertical dashed lines.

\section{APPENDIX B: PROOF OF HERMITIAN SYMMETRY CONDITION FOR ARRAY WEIGHTING}

The purpose of this appendix is to analyze which are the possible phase centers of the combination of the patterns in a uniform linear array. We start by considering the phase center shift of a uniform linear array of $N$ isotropic elements spaced by $d_{a z}$, in the region $[0, L], L=(N-1) \cdot d_{a z}$ as illustrated in Fig. 17. The desired phase center lies at the position $x_{P H}=\alpha \cdot L$, with $0 \leq \alpha \leq 1$. In particular, $x_{P H}=n \cdot d_{a z}$, 
with integer $n$, are trivial cases corresponding to the usage of single elements.

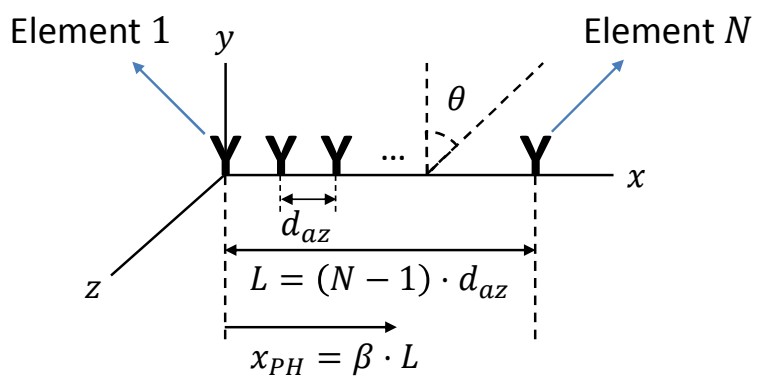

Fig. 17: Geometry of a general $N$-element uniform linear array of inter-element spacing $d_{a z}$ and total distance between outermost elements $L=(N-1) \cdot d_{a z}$. The goal is to obtain a phase center in the intermediate position $x_{P H}=\alpha \cdot L, 0 \leq \alpha \leq 1$ within the array.

The linear combination of the received signals of the antenna array, applying the complex weight $w_{n}$ for the $n^{\text {th }}$ element, yields a resulting far-field pattern. At off-boresight angle $\theta$, for small $\theta$, it can be approximated by the well-known array factor [25],[43]

$A F(\theta)=\sum_{n=0}^{N-1} w_{n} \cdot \exp \left(j \cdot n \cdot \frac{2 \cdot \pi \cdot d_{a z}}{\lambda} \cdot \sin (\theta)\right)$,

where $\lambda$ is the wavelength.

Let the auxiliary spatial frequency be $f_{s p}(\theta)=\sin (\theta) / \lambda$. This quantity is closely related to the Doppler frequency in the case of azimuth. Using it, one may write the conjugate complex of (26) as

$$
\begin{aligned}
A F^{*}\left(f_{s p}\right)= & \sum_{n=0}^{N-1} w_{n}^{*} \cdot \exp \left(-j \cdot 2 \cdot \pi \cdot f_{s p} \cdot\left(n \cdot d_{a z}\right)\right) \\
A F^{*}\left(f_{s p}\right)= & \sum_{n=0}^{N-1} \int_{-\infty}^{\infty} w^{*}(x) \cdot \delta\left(x-n \cdot d_{a z}\right) \\
& \cdot \exp \left(-j \cdot 2 \cdot \pi \cdot f_{s p} \cdot x\right) d x .
\end{aligned}
$$

In the last equation, the summation is changed to an integral by defining the continuous weight function $w(x)$. Taking the complex conjugate and changing the integration variable to $-x$ yields

$$
\begin{aligned}
A F \quad\left(f_{s p}\right)= & \sum_{n=0}^{N-1} \int_{-\infty}^{\infty} w(-x) \cdot \delta\left(-x-n \cdot d_{a z}\right) \\
& \cdot \exp \left(-j \cdot 2 \cdot \pi \cdot f_{s p} \cdot x\right) d x,
\end{aligned}
$$

which is the Fourier Transform of the (theoretical) spacereversed continuous weight distribution $w(-x)$. This distribution is considered to be non-zero only over the interval $-x \in[0,-(N-1) \cdot d]$, and sampled at $x=n \cdot d_{a z}$, i.e. the position of the elements (and thus of their respective phase centers). Clearly (28) may also be written in terms of the Discrete-Time Fourier Transform of the sequence $w[n]=w_{n}$ [25]. This shows that the properties of the array factor rely on those of the Fourier Transform [44].

The desired phase center position is $x_{P H}=\alpha \cdot L$, for $0 \leq \alpha \leq 1$. The array factor is thus expected to take the form
$A F\left(f_{s p}\right) \triangleq \exp \left(j \cdot 2 \cdot \pi \cdot(\alpha \cdot L) \cdot f_{s p}\right) \cdot B\left(f_{s p}\right)$,

where the exponential term is related to the position of the phase center $x_{P H}=\alpha \cdot L$. Using the analogy to time Fourier analysis, $x_{P H}$ represents a "spatial-delay" with respect to the spatial frequency $f_{s p} . B\left(f_{s p}\right)$ is a complex modulation of the phase ramp. (29) can also be understood as factorization of $A F\left(f_{s p}\right)$.

A particular case of interest is the well-known uniform weighting with $w_{n}=1 / N$ in (27), equivalent to

$w(x)=\frac{1}{N}$

which yields [43]

$$
\begin{gathered}
A F_{U N I}\left(f_{s p}\right)=\exp \left(j \cdot 2 \cdot \pi \cdot \frac{(N-1) \cdot d_{a z}}{2} \cdot f_{s p}\right) . \\
\frac{\sin \left(N \cdot \pi \cdot d_{a z} \cdot f_{s p}\right)}{\sin \left(\pi \cdot d_{a z} \cdot f_{s p}\right)} \cdot \frac{1}{N}
\end{gathered}
$$

Comparison to (29) shows that the phase center is located in the position of the geometric center of the array (cf. phase ramp term in (31)). The amplitude is modulated by a factor $B\left(f_{s p}\right)$ which is approximately sinc-like in this case (second term in (31)).

In general, the cases of interest are namely the ones in which $B\left(f_{s p}\right)$ is real for all $f_{s p}$. This avoids phase distortions with respect to the position-induced phase ramp. Comparing (28) and (29) gives

$$
\begin{aligned}
B\left(f_{s p}\right)= & \sum_{n=0}^{N-1} \int_{-\infty}^{\infty} w(-x) \cdot \delta\left(-x-n \cdot d_{a z}\right) \\
& \cdot \exp \left(-j \cdot 2 \cdot \pi \cdot f_{s p} \cdot(x+\alpha \cdot L)\right) d x \\
B\left(f_{s p}\right)= & \sum_{n=0}^{N-1} \int_{-\infty}^{\infty} w\left(-x^{\prime}+\alpha \cdot L\right) \\
\cdot & \delta\left(-x^{\prime}+\alpha \cdot L-n \cdot d_{a z}\right) \\
\cdot & \exp \left(-j \cdot 2 \cdot \pi \cdot f_{s p} \cdot x^{\prime}\right) d x^{\prime} .
\end{aligned}
$$

By using conjugation and time reversal one may also write

$$
\begin{aligned}
B^{*}\left(f_{s p}\right)= & \sum_{n=0}^{N-1} \int_{-\infty}^{\infty} w^{*}\left(x^{\prime}+\alpha \cdot L\right) \\
& \cdot \delta\left(x^{\prime}+\alpha \cdot L-n \cdot d_{a z}\right) \\
& \cdot \exp \left(-j \cdot 2 \cdot \pi \cdot f_{s p} \cdot x^{\prime}\right) d x^{\prime} .
\end{aligned}
$$

A real $B\left(f_{s p}\right)$ requires

$\operatorname{Im}\left\{B\left(f_{s p}\right)\right\}=\frac{B\left(f_{s p}\right)-B^{*}\left(f_{s p}\right)}{2}=0$.

Thus, from (32) and (33), this requires that

$$
\begin{gathered}
\sum_{n=0}^{N-1} \int_{-\infty}^{\infty}\left[w\left(-x^{\prime}+\alpha \cdot L\right) \cdot \delta\left(-x^{\prime}+\alpha \cdot L-n \cdot d_{a z}\right)\right. \\
\left.-w^{*}\left(x^{\prime}+\alpha \cdot L\right) \cdot \delta\left(x^{\prime}+\alpha \cdot L-n \cdot d_{a z}\right)\right] \\
\cdot \exp \left(-j \cdot 2 \cdot \pi \cdot f_{s p} \cdot x^{\prime}\right) d x^{\prime}=0 .
\end{gathered}
$$

To make the integral identically null for all $f_{s p}$, 
$w\left(-x^{\prime}+\alpha \cdot L\right) \cdot \delta\left(-x^{\prime}+\alpha \cdot L-n \cdot d_{a z}\right)=$

$w^{*}\left(x^{\prime}+\alpha \cdot L\right) \cdot \delta\left(x^{\prime}+\alpha \cdot L-n \cdot d_{a z}\right)$

for $n \in[0, N-1]$.

This is a Hermitian symmetry relation [44], applied to the continuous weight distribution with respect to the desired phase center position $x_{P H}=\alpha \cdot L$. Note that the only relevant positions are the "samples" of this distribution at $x_{n}=n \cdot d_{a z}$. These correspond to the positions of the array elements, every other point being merely a mathematical construct. (36) is satisfied, in particular, when

$w\left(-x^{\prime}+\alpha \cdot L\right)=w^{*}\left(x^{\prime}+\alpha \cdot L\right)$,

for all $x^{\prime}$. This condition is sufficient but not necessary, since the points outside $x_{n}=n \cdot d_{a z}$ can take arbitrary values without changing the integrals in (32) and (33). It is however preferred, to simplify the notation.

The relationship between Hermitian symmetry and a real valued transform is indeed a well-known property in Fourier analysis. The implication in this case is that a given phase center position $x_{P H}$ can only be achieved if the weights are Hermitiansymmetric with respect to that point. This also shows that the phase center is always located in the geometrical center of the (sub-)array. The problem of locating the possible phase centers of a planar antenna array (under the "ideal" phase and stable amplitude pattern conditions (29) and (34)) is thus a combinatorial one, since it only depends on the location of the active elements.

\section{REFERENCES}

[1] J. Curlander, R. McDonough, "Synthetic Aperture Radar: Systems and Signal Processing," Jon Wiley \& Sons, 1991.

[2] A. Moreira, P. Prats-Iraola, M. Younis, G. Krieger, I. Hajnsek and K. P. Papathanassiou, "A tutorial on synthetic aperture radar," in IEEE Geoscience and Remote Sensing Magazine, vol. 1, no. 1, pp. 6-43, March 2013.

[3] G. Krieger et al., "Advanced Concepts for Ultra-Wide-Swath SAR Imaging," in 7th European Conference on Synthetic Aperture Radar (EUSAR), Friedrichshafen, pp.1-4, 2008

[4] N. Gebert, G. Krieger and A. Moreira, "Digital Beamforming on Receive: Techniques and Optimization Strategies for High-Resolution Wide-Swath SAR Imaging," IEEE Transactions on Aerospace and Electronic Systems, vol. 45, no. 2, pp.564-592, 2009.

[5] A. Freeman et al., "The "Myth" of the minimum SAR antenna area constraint," IEEE Transactions on Geoscience and Remote Sensing, vol. 38, no. 1, pp. 320-324, Jan 2000.

[6] A. Moreira et al., "Tandem-L: A Highly Innovative Bistatic SAR Mission for Global Observation of Dynamic Processes on the Earth's Surface," IEEE Geoscience and Remote Sensing Magazine, vol. 3, no. 2, pp.8-23, 2015.

[7] I. Sikaneta, C. H. Gierull and D. Cerutti-Maori, "Optimum Signal Processing for Multichannel SAR: With Application to High-Resolution Wide-Swath Imaging," IEEE Transactions on Geoscience and Remote Sensing, vol. 52, no. 10, pp. 6095-6109, 2014.

[8] S. Huber, A. Patyuchenko, G. Krieger and A. Moreira, "Spaceborne Reflector SAR Systems with Digital Beamforming," IEEE Transactions on Aerospace and Electronic Systems, vol. 48, no. 4, pp.3473-3493, 2012.

[9] G. Krieger et al., "SIMO and MIMO System Architectures and Modes for High-Resolution Ultra-Wide-Swath SAR Imaging," in 11th European Conference on Synthetic Aperture Radar (EUSAR), pp. 1-6., Hamburg, 2016.
[10] M. Younis et al., "Techniques and Modes for Multi-Channel SAR Instruments," in 11th European Conference on Synthetic Aperture Radar (EUSAR), pp. 1-6, Hamburg, 2016.

[11] M. Villano, G. Krieger and A. Moreira: "Staggered SAR: High-Resolution Wide-Swath Imaging by Continuous PRI Variation," in IEEE Transactions on Geoscience and Remote Sensing, vol. 52, no.7, pp.4462-4479, 2014.

[12] R. Wang et al., "First Bistatic Demonstration of Digital Beamforming in Elevation With TerraSAR-X as an Illuminator," in IEEE Transactions on Geoscience and Remote Sensing, vol. 54, no. 2, pp. 842-849, Feb. 2016.

[13] G. Krieger, "MIMO-SAR: Opportunities and Pitfalls," in IEEE Transactions on Geoscience and Remote Sensing, vol. 52, no. 5, pp. 2628-2645, May 2014.

[14] M. Suess and W. Wiesbeck, "Side-looking synthetic aperture radar system," European Patent EP 1 241487, filed 2001, published 2002.

[15] S. Huber et al., "Tandem-L: A Technical Perspective on Future Spaceborne SAR Sensors for Earth Observation" submitted to IEEE Transactions on Geoscience and Remote Sensing, 2016.

[16] G. Krieger et al., "Advanced L-Band SAR System Concepts for HighResolution Ultra-Wide-Swath SAR Imaging" in $5^{\text {th }}$ Workshop on Advanced RF Sensors and Remote Sensing Instruments, (ARSI 2017), Noordwijk, 2017.

[17] G. Krieger, et al, "Advanced concepts for high-resolution wide-swath SAR imaging," in 8th European Conference on Synthetic Aperture Radar (EUSAR), pp. 1-4, Aachen, 2010.

[18] M. Villano, "Staggered Synthetic Aperture Radar", Ph.D. dissertation, Institute of Radio Frequency Engineering and Electronics (IHE), Karlsruhe Institute of Technology (KIT), Karlsruhe, Germany, February 2016.

[19] M. Villano, G. Krieger and A. Moreira, "A Novel Processing Strategy for Staggered SAR," in IEEE Geoscience and Remote Sensing Letters, vol. 11, no. 11, pp.1891-1895, 2014.

[20] European Space Agency, (January, 2018) "The CEOS Database: Missions, Instruments and Measurements" [Online]. Available at: http://database.eohandbook.com/database/instrumenttable.aspx

[21] F. Queiroz de Almeida and G. Krieger, "Multichannel Staggered SAR Azimuth Sample Regularization," in 11th European Conference on Synthetic Aperture Radar (EUSAR), pp. 1-6, Hamburg, 2016.

[22] F. Queiroz de Almeida, M. Younis, G. Krieger and A. Moreira, "Staggered SAR Azimuth Processing", in IEEE Transactions on Geoscience and Remote Sensing, vol. 56, issue 3, 2018.

[23] F. Queiroz de Almeida, M. Younis, G. Krieger, F. López-Dekker and A. Moreira, "Synthetik-Apertur-Radarverfahren und Radarvorrichtung," German Patent 102016208899, filed 2016, published 2017.

[24] W. Wang, R. Wang, Y. Deng, T. Balz, F. Hong and W. Xu, "An Improved Processing Scheme of Digital Beam-Forming in Elevation for Reducing Resource Occupation," in IEEE Geoscience and Remote Sensing Letters, vol. 13, no. 3, pp. 309-313, March 2016.

[25] H. L. V. Trees, Optimum Array Processing, New York: John Wiley \& Sons Inc., 2002.

[26] International Telecommunication Union (ITU) (July, 2017) "Recommendation ITU-R RS.577-7 (02/2009): Frequency Bands and Required Bandwidths Used for Spaceborne Active Sensors Operating in the Earth Exploration-Satellite (Active) and Space Research (Active) Services". [Online]. Available at: https://www.itu.int/rec/R-REC-RS.577-7-200902-I/en.

[27] M. Villano, G. Krieger and A. Moreira "Ambiguities and image quality in staggered SAR" in 5th Asia-Pacific Conference on Synthetic Aperture Radar (APSAR), Singapore, pp. 204-209, 2015.

[28] D. D'Aria et al., "A wide swath, full polarimetric, L band spaceborne SAR," in IEEE Radar Conference, Rome, pp. 1-4., 2008.

[29] F. T. Ulaby, C. T. Allen and A. K. Fung, "Method for Retrieving the True Backscattering Coefficient from Measurements with a Real Antenna," in IEEE Transactions on Geoscience and Remote Sensing, vol. GE-21, no. 3, pp. 308313, 1983.

[30] M. Younis, P. López-Dekker and G. Krieger, "Signal and noise considerations in multi-channel SAR" in 16th International Radar Symposium (IRS), Dresden, pp. 434-439., 2015.

[31] TICRA, "GRASP Software", Copenhagen, Denmark. Available: http://www.ticra.com/products/software/grasp. 
[32] L. Lei, G. Zhang and R. J. Doviak, "Bias Correction for Polarimetric PhasedArray Radar With Idealized Aperture and Patch Antenna Elements," in IEEE Transactions on Geoscience and Remote Sensing, vol. 51, no. 1, pp. 473-486, Jan. 2013.

[33] A. Patyuchenko, T. Rommel, P. Laskowski, M. Younis, and G. Krieger, "Digital Beam-Forming Reconfigurable Radar System Demonstrator," in IEEE International Geoscience and Remote Sensing Symposium (IGARSS), pp. 1541-1544, Munich, 2012.

[34] T. Rommel, A. Patyuchenko, P. Laskowski, M. Younis and G. Krieger, "Development of a MIMO Radar System demonstrator - Calibration and demonstration of first results," in 13th International Radar Symposium (IRS), pp. 113-118, Warsaw, 2012.

[35] S. Huber, T. Rommel, A. Patyuchenko and P. Laskowski, "A Reflector Based Digital Beamforming Demonstrator," in 10th European Conference on Synthetic Aperture Radar (EUSAR), pp. 1-4, Berlin, 2014.

[36] S. Bertl, P. Lopez-Dekker, M. Younis and G. Krieger, "Along-track SAR interferometry using a single reflector antenna," IET Radar Sonar \& Navigation, vol. 9, no. 8, pp. 942 - 947, October 2015.

[37] M. Villano, G. Krieger and A. Moreira, "An Analytical Phase-Only Beam Shaping Method for High-Resolution Wide-Swath SAR" submitted to IEEE Geoscience and Remote Sensing Letters, 2016.

[38] European Space Agency, “Sentinel-1 ESA's Radar Observatory Mission for GMeS Operational Services", Report SP-1322/1, 2012. Available at: https://sentinel.esa.int/documents/247904/349449/S1 SP-1322_.pdf, 2017.

[39] Canadian Space Agency (October, 2017), "RADARSAT: Satellite Characteristics", [Online]. Available at: http://www.asccsa.gc.ca/eng/satellites/radarsat/radarsat-tableau.asp, 2017.

[40] M. Younis, S. Huber, A. Patyuchenko, F. Bordoni and G. Krieger, "Performance Comparison of Reflector- and Planar-Antenna Based Digital Beam-Forming SAR," International Journal of Antennas and Propagation, vol. 2009, Article ID 614931, 13 pages, 2009.

[41] C. C. Lin et al., "ESA's SAR Technology and Future Mission Concept Developments beyond Sentinel-1," in 7th European Conference on Synthetic Aperture Radar, pp. 14, Friedrichshafen, Germany, 2008.

[42] G. Krieger et al., "In-Orbit Relative Amplitude and Phase Antenna Pattern Calibration for Tandem-L," in 12th European Conference on Synthetic Aperture Radar, pp. 14, Aachen, Germany, 2018.

[43] C. Balanis, "Arrays: Linear, Planar, and Circular," in Antenna Theory: Analysis and Design, New York: John Wiley \& Sons Inc., 1997.

[44] A. Papoulis, The Fourier Integral and its Applications, New York: McGrawHill Co., 1962.

[45] J. Liu et al., "Adaptive beamforming with sidelobe control: a second-order cone programming approach," in IEEE Signal Processing Letters, vol. 10, no. 11, pp. 331-334, Nov. 2003

[46] M. S. Andersen, J. Dahl, and L. Vandenberghe, "CVXOPT: A Python package for convex optimization", version 1.1.8. Available at cvxopt.org, 2016.

[47] G. Sagnol, et al, "PICOS: A Python Interface for Conic Optimization Solvers", version 1.1.2. Available at picos.zib.de, 2016. 